\title{
Crossed graphene nanoribbons as beam splitters and mirrors for electron quantum optics
}

\author{
Sofia Sanz $\odot,{ }^{1, *}$ Pedro Brandimarte, ${ }^{1}$ Géza Giedke $\odot,{ }^{1,2}$ Daniel Sánchez-Portal,${ }^{3}$ and Thomas Frederiksen $\oplus^{1,2, \dagger}$ \\ ${ }^{1}$ Donostia International Physics Center (DIPC), Paseo Manuel de Lardizabal 4, E-20018 Donostia-San Sebastián, Spain \\ ${ }^{2}$ IKERBASQUE, Basque Foundation for Science, E-48013 Bilbao, Spain \\ ${ }^{3}$ Centro de Física de Materiales (CFM) CSIC-UPV/EHU, Paseo Manuel de Lardizabal 5, E-20018 Donostia-San Sebastián, Spain
}

(Received 23 May 2020; accepted 8 July 2020; published 24 July 2020)

\begin{abstract}
We analyze theoretically four-terminal electronic devices composed of two crossed graphene nanoribbons (GNRs) and show that they can function as beam splitters or mirrors. These features are identified for electrons in the low-energy region where a single valence or conduction band is present. Our modeling is based on $p_{z}$ orbital tight binding with Slater-Koster-type matrix elements fitted to accurately reproduce the low-energy bands from density functional theory calculations. We analyze systematically all devices that can be constructed with either zigzag or armchair GNRs in AA and AB stackings. From Green's function theory the elastic electron transport properties are quantified as a function of the ribbon width. We find that devices composed of relatively narrow zigzag GNRs and AA-stacked armchair GNRs are the most interesting candidates to realize electron beam splitters with a close to 50:50 ratio in the two outgoing terminals. Structures with wider ribbons instead provide electron mirrors, where the electron wave is mostly transferred into the outgoing terminal of the other ribbon, or electron filters where the scattering depends sensitively on the wavelength of the propagating electron. We also test the robustness of these transport properties against variations in the intersection angle, stacking pattern, lattice deformation (uniaxial strain), inter-GNR separation, and electrostatic potential differences between the layers. These generic features show that GNRs are interesting basic components to construct electronic quantum optical setups.
\end{abstract}

DOI: 10.1103/PhysRevB.102.035436

\section{INTRODUCTION}

The similarities between the wave nature of electrons propagating coherently in ballistic conductors with photon propagation in optical waveguides has spawned the field of electron quantum optics $[1,2]$. In this way several electronic analogs of optical setups—-such as the Mach-Zehnder [3,4] and Fabry-Pérot [5-7] interferometers, as well as the Hanbury Brown-Twiss [8-11] geometry to study the Fermion antibunching and the two-particle Aharonov-Bohm [12] effectshave been implemented. Fundamental components for these setups include mirrors (Ms), beam splitters (BSs, i.e., partially transparent mirrors), and wavelength filters. Such control elements for electron beams are important in the fields of quantum information and solid-state quantum computation: By sending a single electron through a BS one can generate a mode-entangled state that can be used to violate a Bell inequality [13] or for quantum teleportation [14,15]. A BS is the central building block of the Hong-Ou-Mandel setup to test the indistinguishability [16] or the entanglement [17] of electrons entering in the two input ports. With two BSs and two oriented Ms the Mach-Zehnder interferometer can be fully implemented, which has been demonstrated to work as a quantum logic processor [18].

A platform with remarkable prospects for electron quantum optics are graphene-based systems, in which several

\footnotetext{
*sofia.sanz@dipc.org

†thomas_frederiksen@ehu.eus
}

pioneering experiments on electron beam splitters and related devices have been performed [19,20]. More recently, graphene nanoribbons (GNRs) [21,22] have emerged as attractive candidates for the construction of molecular-scale electronic devices [23] because they inherit some of the exceptional properties from graphene while having tunable electronic properties, such as the opening of a band gap depending on their width and edge topology [24-28]. The electron coherence length in GNRs can be long, with values of the order $\sim 100 \mathrm{~nm}$ being reported [29-31]. Furthermore, ballistic transport can be rather insensitive to edge defects because of the presence of localized edge states (e.g., in zigzag GNRs) and the dominating Dirac-like physics, that make the current flow maximally through the center of the ribbon [32]. With the advent of bottom-up fabrication techniques, long defect-free samples can be chemically synthesized with both armchair (AGNR) [33] and zigzag (ZGNR) [34] edge topologies via on-surface synthesis. Manipulation of GNRs with scanning tunneling probes has been also demonstrated $[35,36]$, opening the possibility to build two-dimensional multiterminal graphene-based electronic circuits [37-41].

Recently, it has been shown theoretically that two crossed GNRs with a relative angle of $60^{\circ}$ can behave as a BS for valence- and conduction-band electrons [42,43], since such four-terminal devices were found to divide the electron beam into two out of the four arms with an equal transmission probability of $50 \%$. In this paper we analyze this possibility more generally and show that all the mentioned beam-control elements (BS, M, filters) can be realized with a suitable choice of two crossed GNRs. More specifically, we compute 
the electron transport properties of these devices in terms of the edge topology and width of the GNRs, and the precise alignment and stacking of the ribbons.

The problem is theoretically approached by means of tight-binding (TB) modeling, which is known to reproduce graphitelike systems with sufficient accuracy [44-48] while allowing one to explore a large number of systems of considerable sizes in a fast and transparent way. For instance, the geometry of a crossing between two 50-atom-wide GNRs readily comprises around 10000 atoms. The main complexity of the modeling lies in the description of the interlayer couplings, for which we use a Slater-Koster parametrization [49] that has proven successful for describing the band structure and velocity renormalization of Dirac electrons in twisted bilayer graphene [50,51]. The employed technique can describe arbitrary device geometries and therefore allows us to also study the robustness of the predicted transport properties against variations in the intersection angle, stacking pattern, lattice deformation (uniaxial strain), inter-GNR separation, and electrostatic potential differences between the layers. With this, we give a complete analysis of the transport properties of crossed GNRs, highlight their tunability, and provide quantitative data that can serve as a guide for design optimization.

This paper is organized as follows: In Sec. II we introduce the general TB Hamiltonian used to describe the kinetics of the electrons traveling through the different devices as well as the scattering formalism used to compute transmission and reflection probabilities of incoming electron waves from the different leads. In Sec. III we present a complete analysis of the transport properties based on the key combinations of stacking pattern, edge topology, and width of the GNRs. Finally, the conclusions and remarks are provided in Sec. IV.

\section{METHODOLOGY}

The general setup of this study, illustrated in Fig. 1(a), comprises two infinite GNRs crossed with a relative angle $\theta=$ $60^{\circ}$ (see Sec. III A for a discussion of this choice of angle). The scattering region (intersection) breaks the translational invariance of the infinite ribbons, for which we will use the Green's function formalism to solve the Schrödinger equation with open boundary conditions.

The system is divided into the device (scattering) region that contains the intersection between the two ribbons, and the four semi-infinite GNRs (periodic electrodes), represented as red rectangles in Fig. 1(a). The total Hamiltonian is correspondingly split into the different parts

$$
H_{T}=H_{d}+\sum_{\alpha}\left(H_{\alpha}+H_{\alpha d}\right)
$$

where $H_{d}$ is the device Hamiltonian, $H_{\alpha}$ the $\alpha$-electrode Hamiltonian, and $H_{\alpha d}$ the coupling between these two subsystems.

\section{A. Tight-binding Hamiltonian}

The use of a local basis in combination with Green's function techniques provides an efficient way for obtaining the transport properties in terms of microscopic parameters.

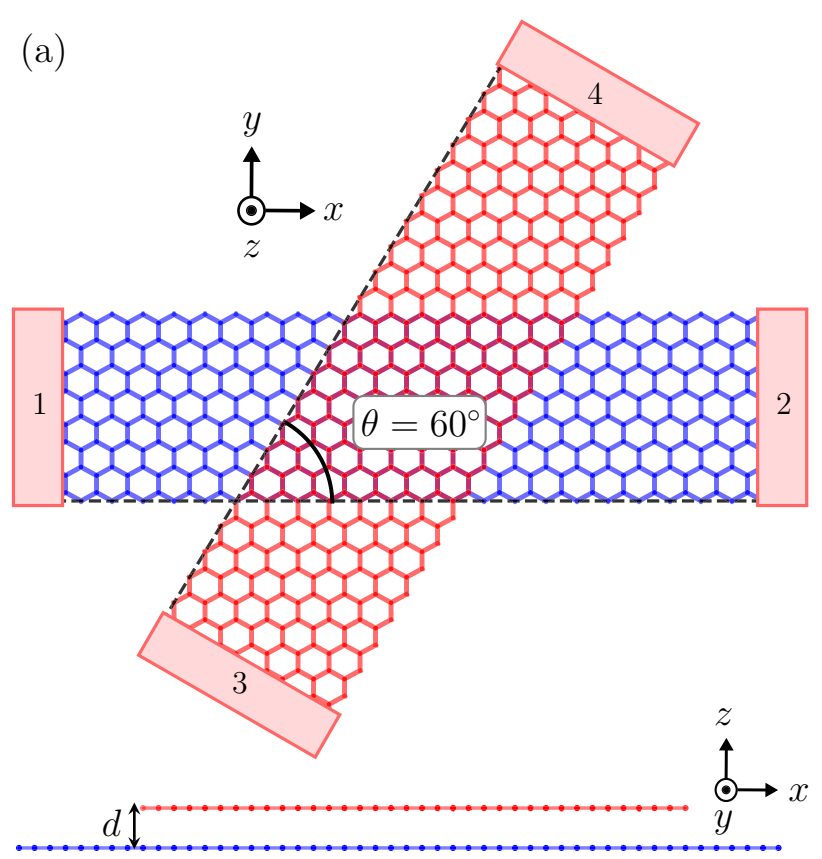

(b)

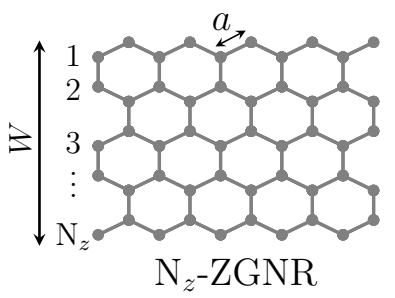

(c)

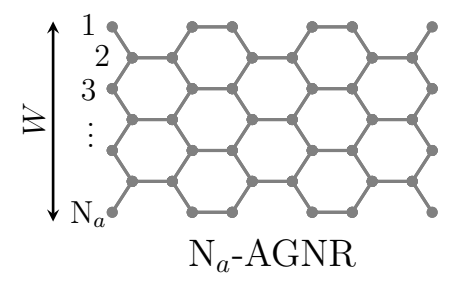

FIG. 1. Illustration of the general setup. (a) A four-terminal device is formed by two crossed 8 -ZGNRs with a relative angle $\theta=60^{\circ}$. The bottom (top) ribbon is drawn in blue (red) with carbon atoms at each vertex. The four semi-infinite leads, numbered $1-4$, are attached in the contact regions represented with red rectangles. The ribbons lie out of plane separated by a distance $d$ along the $z$ axis (see side view). Definition of the width $W$ of (b) ZGNRs and (c) AGNR in terms of the number of carbon atoms $N$ across the ribbon. The interatomic distance is denoted by $a$.

We write the single-particle TB Hamiltonian in an orthogonal basis as

$$
H=\sum_{i} \epsilon_{i} c_{i}^{\dagger} c_{i}+\sum_{i j} t_{i j}\left(c_{i}^{\dagger} c_{j}+\text { H.c. }\right)
$$

where $c_{i}^{\dagger}\left(c_{i}\right)$ creates (annihilates) an electron on site $i$ with energy $\epsilon_{i}$. We further define the Fermi level as $E_{F}=\epsilon_{i}$, corresponding to half-filled carbon $p_{z}$ orbitals. The matrix element $t_{i j}$ between orbitals $i$ and $j$ is described by SlaterKoster-type two-center $\pi$ and $\sigma$ bond integrals between two $p_{z}$ atomic orbitals [49]

$$
t_{i j}=V_{p p \pi}\left(1-l^{2}\right)+V_{p p \sigma} l^{2},
$$

where $l$ is the cosine of the angle formed between the distance vector $\hat{r}_{i j}$ for the $i j$ atom pair and the unit vector that defines 
the $z$ direction [cf. Fig. 1(a)], i.e., $l=\hat{r}_{i j} \cdot \hat{e}_{z} /\left|r_{i j}\right|$. The twocenter integrals are expressed as

$$
\begin{aligned}
& V_{p p \pi}=-t_{\|} e^{q_{\pi}\left(1-\frac{\left|r_{i j}\right|}{a}\right)}, \\
& V_{p p \sigma}=-t_{\perp} e^{q_{\sigma}\left(1-\frac{\left|r_{i j}\right|}{d}\right)},
\end{aligned}
$$

where $t_{\|}\left(t_{\perp}\right)$ is the intra-GNR (inter-GNR) hopping parameter between atoms separated by the interatomic (interlayer) distance fixed to $a=1.42 \AA(d=3.34 \AA)$ in our model (see Fig. 1). The decay rates of the bond integrals with the atomic separation, $q_{\sigma}$ and $q_{\pi}$, are isotropic and therefore related by $q_{\sigma} / d=q_{\pi} / a$. This model, characterized by $t_{\|}, t_{\perp}$ and the decay rate (which can be determined by fixing the second-nearest-neighbor coupling), successfully describes $\pi$ electrons in twisted bilayer graphene [51]. However, it does not capture many-body effects such as, e.g., the difference in the nearest-neighbor hopping parameter for different lattice sites as in the Slonczewski-Weiss-McClure (SWM) model for graphite [45,52-54].

In this work we use $t_{\|}=2.682 \mathrm{eV}$ and $t_{\perp}=0.371 \mathrm{eV}$. For the third model parameter we refer to the in-plane nextnearest-neighbor matrix element $t^{\prime}=0.0027 \mathrm{eV}$. These parameters were obtained by fitting to the low-energy band structure of AB-stacked bilayer graphene simulated with SIESTA [55] as explained in the Appendix. The satisfactory agreement between TB and density functional theory (DFT) (Fig. 14) further justifies that, at least for our purposes, manybody effects such as in the SWM model can be neglected.

\section{B. Transport calculations}

In order to perform transport calculations we use the nonequilibrium Green's function (NEGF) method [56-58]. In particular, to obtain the transmission probabilities $\left(T_{\alpha \beta}\right)$ between the different pairs of electrodes $(\alpha \neq \beta)$, we use the Landauer-Büttiker formula [59],

$$
T_{\alpha \beta}=\operatorname{Tr}\left[\Gamma_{\alpha} G \Gamma_{\beta} G^{\dagger}\right], \quad \alpha \neq \beta,
$$

where $\Gamma_{\alpha}=i\left(\Sigma_{\alpha}-\Sigma_{\alpha}^{\dagger}\right)$ is the broadening matrix, related to the non-Hermitian part of the retarded electrode self-energy $\Sigma_{\alpha}$, due to the coupling of the $\alpha$ th semi-infinite lead to the scattering center and $\alpha, \beta=1, \ldots, 4$ (cf. Fig. 1). Further,

$$
G_{d}=\left(\mathbb{I} E-H_{d}-\sum_{\alpha} \Sigma_{\alpha}\right)^{-1}
$$

is the retarded Green's function of the device region and $\mathbb{I}$ the identity matrix (orthogonal basis). The dependency on the electron energy $E$ of these key quantities is implicit.

The reflection probability $\left(T_{\alpha \alpha}=R_{\alpha}\right)$ can be conveniently written as a difference between the bulk electrode transmission $M_{\alpha}$ (i.e., the number of open channels/modes in electrode $\alpha$ at a given energy) and the scattered part into the other electrodes $\left(\sum_{\beta} T_{\alpha \beta}\right)$ as

$$
R_{\alpha}=M_{\alpha}-\sum_{\beta \neq \alpha} T_{\alpha \beta} .
$$

From Eq. (7) we can also obtain the spectral function $A_{\alpha}$ for states coupled to electrode $\alpha$,

$$
A_{\alpha}=G \Gamma_{\alpha} G^{\dagger} .
$$

The diagonal elements $A_{\alpha}(i, i) / 2 \pi$ correspond to the local density of states (DOS) at sites $i$ of the scattering states originating from electrode $\alpha$.

Computationally, we constructed the Hamiltonian matrix with the SISL package $[60,61]$ and computed transmission probabilities and spectral DOS with TBTRANS [61].

\section{RESULTS}

In this section we present results for the electron transport properties through an extensive set of four-terminal devices formed by two crossed ribbons. We analyze the role of the precise stacking and alignment of the crossing area for both ZGNR- and AGNR-based devices in all their possible configurations.

\section{A. Possible device configurations}

The symmetry of the honeycomb lattice yields a perfect matching between the bottom and top GNR lattices for $\theta=$ $60^{\circ}$. In this situation it is expected that the maximized interlayer coupling enhances the transfer of electrons between the ribbons, as shown in Refs. [41-43]. In Fig. S1 in the Supplemental Material (SM) [62] we performed transport calculations for crossed 8-ZGNRs both in the AA and AB stackings as a function of the crossing angle between the GNRs, where such behavior is observed for angles approaching $60^{\circ}$. We therefore focus the discussion on devices formed by crossed GNRs with an intersecting angle of $\theta=60^{\circ}$. However, the inter-GNR transmission is also enhanced for angles within $\left[50^{\circ}, 70^{\circ}\right]$, which highlights the tunability of our devices. Note that experiments on twisted bilayer graphene report that the rotation angle between the layers can be precisely controlled down to fractions of a degree $\left(0.01^{\circ}\right)$ [63-65].

For a systematic analysis we begin by considering all the possible devices that can be built with two crossed AA- or ABstacked GNRs with a relative angle of $60^{\circ}$. These are sketched Fig. 2. In the case of crossed ZGNRs there exist two configurations, the $\mathrm{AB}$ stacking [labeled $\mathrm{AB}$, Fig. 2(a)] and the AA stacking [labeled AA, Fig. 2(b)]. These two geometries have different symmetries, indicated by the reflection planes (dashed lines) in Fig. 2. While $\mathrm{AB}$ has only one reflection symmetry, AA has two. Here, and in the following, we refer only to symmetries in the $x y$ plane. The additional operation of reflection in the $z$ direction to interchange the top and bottom ribbon is physically not important and therefore implicit.

In the case of AGNRs there are two different AA-stacked configurations [labeled AA-1 and AA-2, Figs. 2(c) and 2(d)], as well as two different $\mathrm{AB}$-stacked configurations [labeled AB-1 and AB-2, Figs. 2(e) and 2(f)]. For instance, starting from AA-1, one can obtain AA-2 by translating the upper (red) ribbon by $-\sqrt{3} a \hat{y}$ with respect to the lower one. Similarly, AB-1 (AB-2) can be obtained from AA-1 by translating the upper (red) ribbon by $+a \hat{x}(-a \hat{x})$ with respect to the lower one. Again, these four generic configurations have different symmetries as indicated in Figs. 2(c)-2(f). 

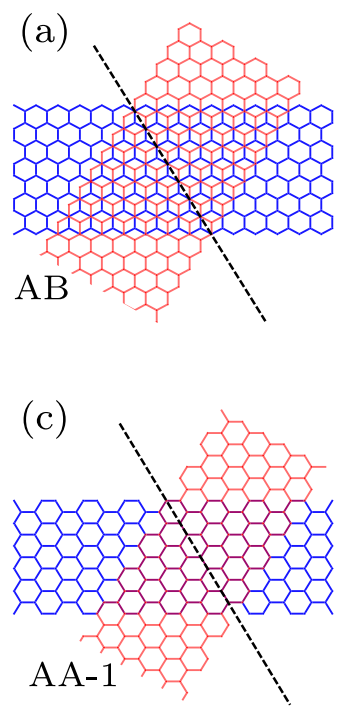

(e)

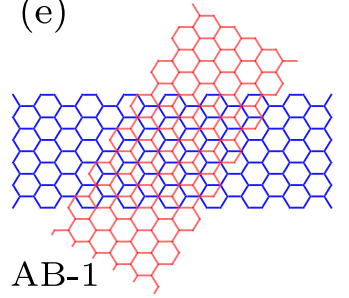

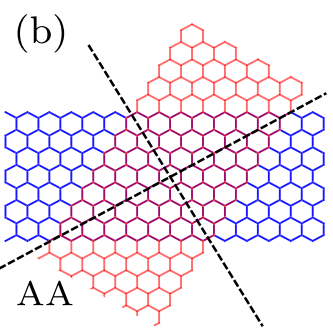

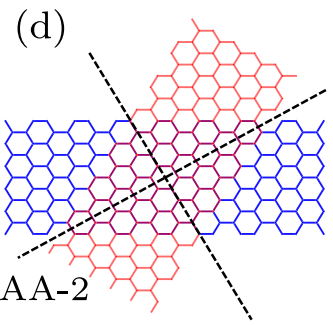

(f)

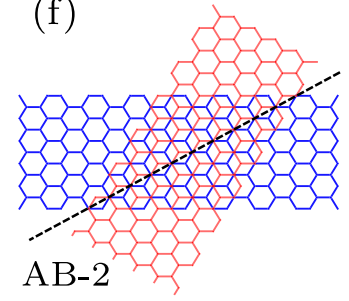

FIG. 2. Geometries of the different stackings that can be constructed from the crossing of two GNRs with a relative angle of $60^{\circ}$. The bottom (top) ribbon is drawn in blue (red) with carbon atoms at each vertex. For ZGNR-based devices there exists only one AA- and one AB-stacked configuration, labeled (a) $\mathrm{AB}$ and (b) AA (exemplified here by 8-ZGNR). For AGNR-based devices there exist two AA- and two AB-stacked configurations, labeled (c) AA-1, (d) AA-2, (e) AB-1, and (f) AB-2 (exemplified here by 11-AGNR). The dashed lines show the symmetry (reflection) planes that preserve the Hamiltonian of each crossing when such operation is applied to them.

The reflection planes imply that there are operations which leave the scattering potential (created by the intersection of the two ribbons) unchanged. This is, if we apply one or more reflections across the indicated axes, the Hamiltonian of the new device does not change. Consequently, the Green's function, and all the transport properties derived from it, will also remain unchanged under some particular electrode permutations.

Let us begin by discussing the properties of these six different configurations with particular examples constructed from 8-ZGNRs and 11-AGNRs. In Fig. 3 we show the spectral DOS of scattering electrons that come in from electrode $\alpha=1$ as obtained from Eq. (9) for each configuration at specific energies. In this real-space representation it is easy to see where the scattered electron wave propagates after being injected into the device. The large DOS that appears in the input electrode region does not correspond to the backscattered electrons, but rather to the DOS of the incoming electrons (as we will show later on). This is also illustrated in Fig. S2 [62],
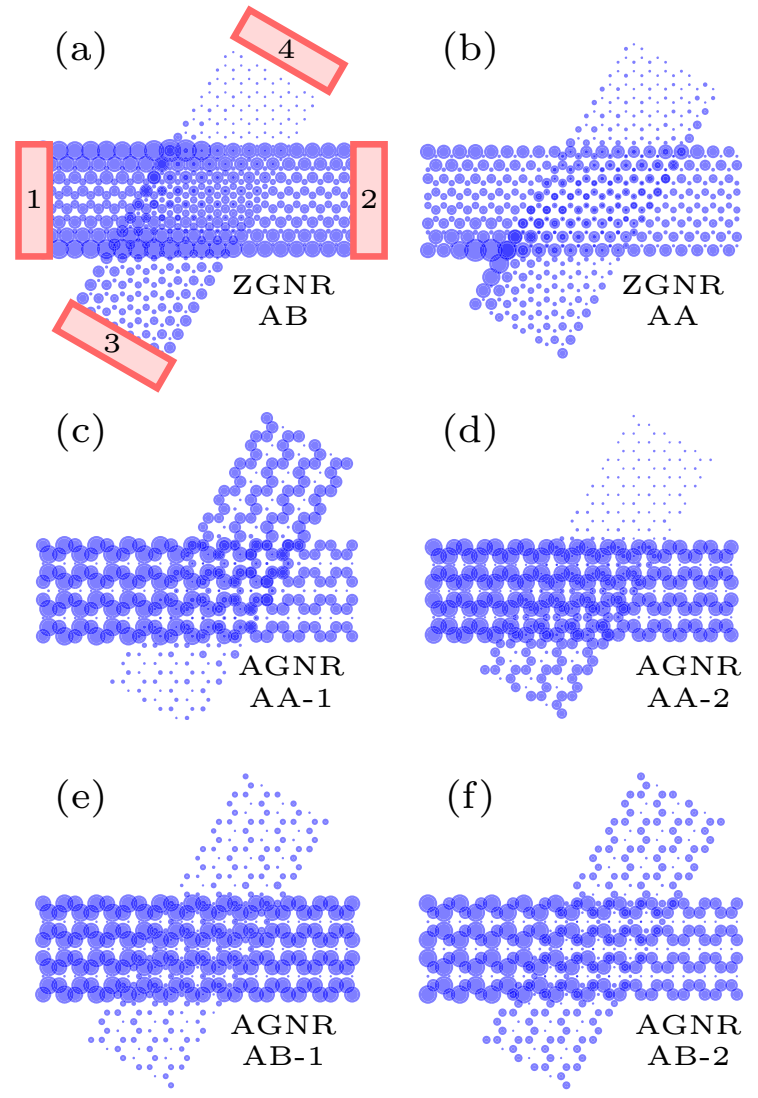

FIG. 3. Spectral DOS of scattering electrons incoming from electrode $\alpha=1$ obtained from Eq. (9), for the specific geometries defined in Fig. 2: (a) 8-ZGNR AB, (b) 8-ZGNR AA, (c) 11-AGNR AA-1, (d) 11-AGNR AA-2, (e) 11-AGNR AB-1, and (f) 11-AGNR AB-2. The spectral DOS were calculated at $E=200 \mathrm{meV}$ for ZGNRs and at $E=0 \mathrm{meV}$ for AGNRs.

where we complement the results shown in Fig. 3 by plotting the bond currents between nearest-neighbor atoms, where the arrows indicate the direction of the flowing electrons.

For the ZGNR devices, Figs. 3(a) and 3(b) and Figs. S2(a) and S2(b) show that an electron injected from $\alpha=1$ in both cases only escapes from the scattering center into electrodes $\beta=2,3$, i.e., terminals 1 and 4 are suppressed. This lack of backscattering (and preferential scattering into only one of the two arms of the other ribbon) is a very general and robust feature for ZGNRs which holds for different widths, stackings, and energies, and it is instrumental for the applications we have in mind. An explanation, supported by continuum-model calculations [66,67], is the valley (chirality) preservation in low-energy bands of ZGNRs. For the two AA-stacked AGNR devices, Figs. 3(c) and 3(d) and Figs. S2(c) and S2(d) show that the outgoing terminals $\beta=1$ and $\beta=3(\beta=4)$ for AA-1 (AA-2) are suppressed. These two cases are interesting since their relative displacement of only $\sqrt{3} a \hat{y}$ leads to very different electron transport: For AA-1 the split electron turns by $60^{\circ}$, while for AA-2 the bend is $120^{\circ}$. Unlike for ZGNR devices, the suppression of two terminals is not general for all AGNR widths, and rather depends on the AGNR family, as shown in Figs. S5-S16 [62]. In the case of the two AB-stacked 
ribbons, Figs. 3(e) and 3(f) show that an electron wave in these devices is scattered qualitatively (yet not quantitatively) similarly and into all outgoing electrodes.

\section{B. Symmetry considerations}

Since we deal with four-terminal devices, the matrix of transmission and reflection probabilities, Eqs. (6) and (8), has the general form

$$
\mathbf{T}=\left(\begin{array}{llll}
R_{1} & T_{12} & T_{13} & T_{14} \\
T_{21} & R_{2} & T_{23} & T_{24} \\
T_{31} & T_{32} & R_{3} & T_{34} \\
T_{41} & T_{42} & T_{43} & R_{4}
\end{array}\right) .
$$

However, due to symmetries there are not 16 independent quantities in this matrix. First, in the absence of a magnetic field, time reversal symmetry forces $T_{\alpha \beta}=T_{\beta \alpha}$. This reduces the matrix to ten independent elements, e.g., those without the dark gray background $(\alpha>\beta)$ in Eq. (10). Second, the symmetries indicated in Fig. 2 reduce the number of independent elements of the matrix further. The reflection plane $y=\sin \left(-60^{\circ}\right) x$ maps the electrode labels $(1,2,3,4) \leftrightarrow$ $(4,3,2,1)$ with unchanged transmissions, e.g., which allows us to consider $R_{3}, R_{4}, T_{24}$, and $T_{34}$ as dependent variables [four of the light gray elements in Eq. (10)]. Similarly, the reflection plane $y=\sin \left(30^{\circ}\right) x$ implies $(1,2,3,4) \leftrightarrow(3,4,1,2)$ and $R_{3}$, $R_{4}, T_{23}$, and $T_{34}$ as possible dependent variables (four of the light gray elements). The combination of both reflection planes further implies $(1,2,3,4) \leftrightarrow(2,1,4,3)$ and $R_{2}$ and $T_{23}$ as further dependent variables (i.e., all gray elements in this case). In summary, depending on the number of symmetries, the transmission probabilities of any given device will be fully characterized by either four, six, or ten independent matrix elements.

Figure 4 shows the full, energy-resolved transmission matrix [Eq. (10)] obtained numerically for devices formed of two crossed ZGNRs in the $\mathrm{AB}$ configuration for a range of different ribbon widths $W$. As ZGNR AB displays only one reflection plane, the transmission probabilities for these systems are, in principle, characterized by six independent quantities. However, qualitatively only four independent ones are readily identified in Fig. 4. Only upon close inspection of the data do all the expected differences emerge. The reason for the seemingly higher symmetry (corresponding to two reflection planes) is the fact that the scattering potential created by the crossings between the GNRs depends exponentially on the atomic distances between the GNRs, and therefore is dominated by the closest atom pairs. These atom pairs, shown in Fig. S3(a) [62], are in fact symmetric with respect to both reflection planes.

More generally, for all the configurations in Fig. 2 we find that the scattering potentials are dominated by terms with at least one reflection plane (Fig. S3). For all practical purposes, the effective symmetry appears higher and it suffices to describe the transmission probabilities with only four or six independent quantities.

In the following we will thus only consider it sufficient to discuss electrons incoming from terminal $\alpha=1$. However, for

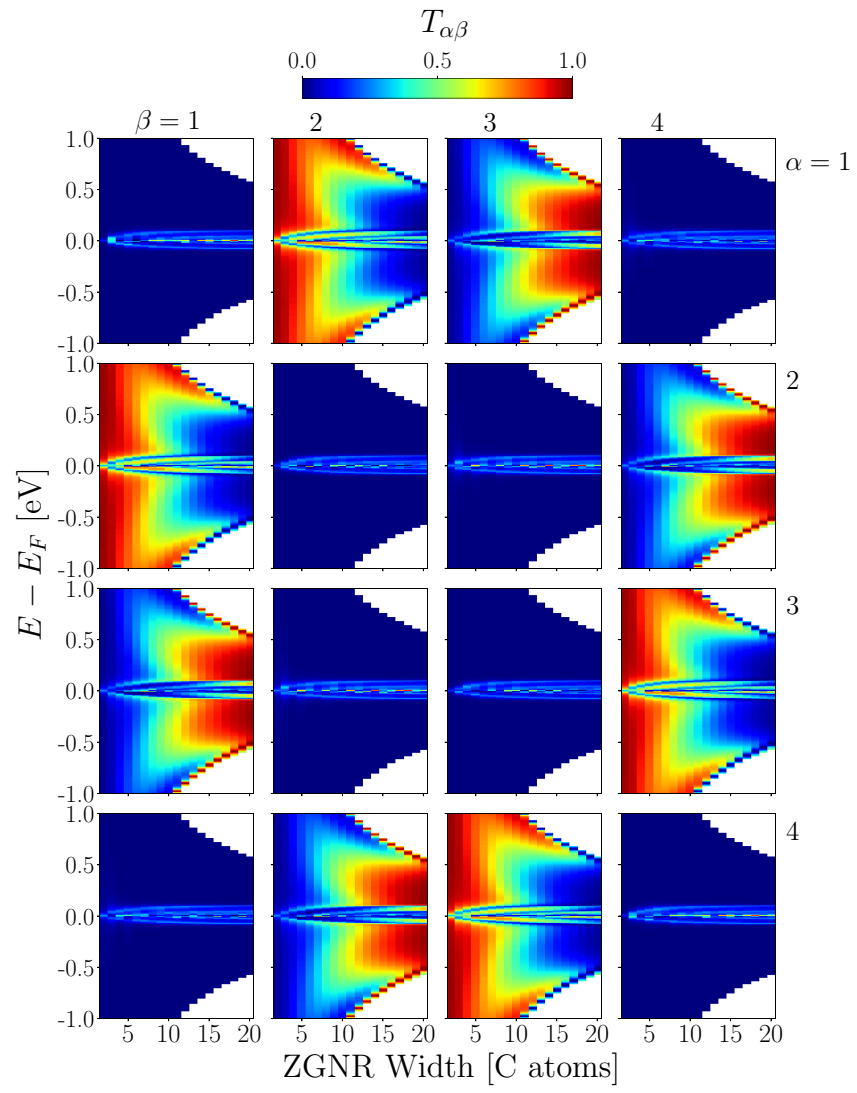

FIG. 4. Full transmission probability matrix $T_{\alpha \beta}$ between all the electrode pairs for ZGNRs crossed in the $\mathrm{AB}$ configuration as a function of the ribbon width $W$ and electron energy $E$. Only data for the first subband are shown (white regions correspond to multiple electronic bands in the ribbons).

completeness we show the full transmission matrices for all the considered systems in Figs. S4-S16 [62].

\section{Beam splitters and mirrors}

Looking again at Fig. 4 and focusing on the first row (electron beam injected from terminal $\alpha=1$ ), we observe distinct regimes where the devices would present particular electron quantum optical characteristics. We are especially interested in geometries for which the transmission matrix allows us to designate two input and two output electrodes in the sense that any electron sent in through one of the input ports is scattered predominantly into the two output ports with very little reflection or transmission into the other input. For instance, the green areas in the plots show where the device behaves as a BS, since they show that the electron beam is scattered only into two out of the four possible arms with a transmission probability that lies around $T_{12} \sim T_{13} \sim 0.5$. One can also identify regimes in which the device can work as a $\mathrm{M}$ where $T_{13} \sim 1$. This situation corresponds to the red areas in Fig. 4, since the electron would enter from terminal $\alpha=1$ and turn $120^{\circ}$ to go out exclusively into terminal $\beta=3$ with low reflection. The energy dependence of the transmission functions is very symmetric with respect to the Fermi level, reflecting the approximate particle-hole symmetry characteristic 
of a half-filled bipartite lattice. Nevertheless, the presence of next-nearest-neighbor couplings in our TB model in principle breaks this symmetry.

On one hand, we note that for energies close to the Fermi level $\left(\left|E-E_{F}\right|<0.07 \mathrm{eV}\right)$ in Fig. 4, the electron is scattered into all four output ports, which makes this small energy window not very interesting for electron quantum optical purposes. These features probably arise due to the hybridization of states from the flat bands of the individual ribbons in the overlapping area. The band structures for both monolayer and bilayer ZGNRs are shown in Fig. S17 [62]. On the other hand, we note here that outside the low-energy region (where there is more than one electronic band) we find for all systems that reflection and interband scattering play a larger role in the electron transport through these devices, as the number of open channels (modes) grows with energy. In other words, it was not possible to identify conditions for realizing BS or M at energies with multiple subbands in the GNRs. Therefore the following discussion is focused on the energy window corresponding to a single (conduction or valence) band, since the most interesting physics related to the electron quantum optical features were identified here.

\section{Quality of the realized mirrors and beam splitters}

To obtain a qualitative picture across all the possible systems of the most suitable candidates for BSs or Ms, we construct in the following a figure of merit (FM). On the one hand, we look for candidate systems where a significant part of the scattered electron wave can be transferred to the other ribbon, i.e., that $T_{13}$ or $T_{14}$ is large. We encode this property in the quantity $\tau \equiv \max \left(T_{13}, T_{14}\right)$. On the other hand, for a suitable $\mathrm{BS}$ or $\mathrm{M}$ it is important that the reflection and transmission to a third electrode should be small. This property is encoded as a "loss" function $\lambda \equiv R_{1}+\min \left(T_{12}, T_{13}, T_{14}\right)$.

Our FM is then defined as

$$
\mathrm{FM}=e^{-20 \lambda} \tanh \left[\frac{1}{20}\left(\frac{1}{|\tau-1|}-\frac{1}{|\tau-1 / 2|}\right)\right] .
$$

We use a linear color scale where BSs $(F M=-1)$ appear as black, M's $(F M=1)$ as red, and uninteresting systems $(F M=0)$ as white. We set $F M$ equal to zero whenever there is more than one band per GNR at the energy considered (as it happens, e.g., for large values of $\left.\left|E-E_{F}\right|\right)$. In that case the sum of all transmission probabilities is equal to the number of bands and thus larger than 1 . This case is not useful for the devices we have in mind, though a more careful analysis may show how to also use the systems in this energy range. In other words, $\lambda$ determines the intensity of the plots while $\tau$ sets the color. The FM is chosen to be highly selective: It decays to about $1 / 2$ of the maximum value for losses (=transmission probability into the undesired output ports) of about 3\%. Similarly, the FM of a loss-free, but unbalanced, $\mathrm{BS}$ is reduced to $\mathrm{FM}=-1 / 2$ at an imbalance of about 57:43. Figures 5 and 6 show the FM for ZGNRs and AGNRs from the metallic $3 p+2$ family, respectively, as a function of electron energy and ribbon width $W$. Overall, these figures show that the most interesting systems are those composed by ZGNRs or AA-stacked AGNRs. For both types of GNRs one can find devices that behave as BS or as M, respectively. For

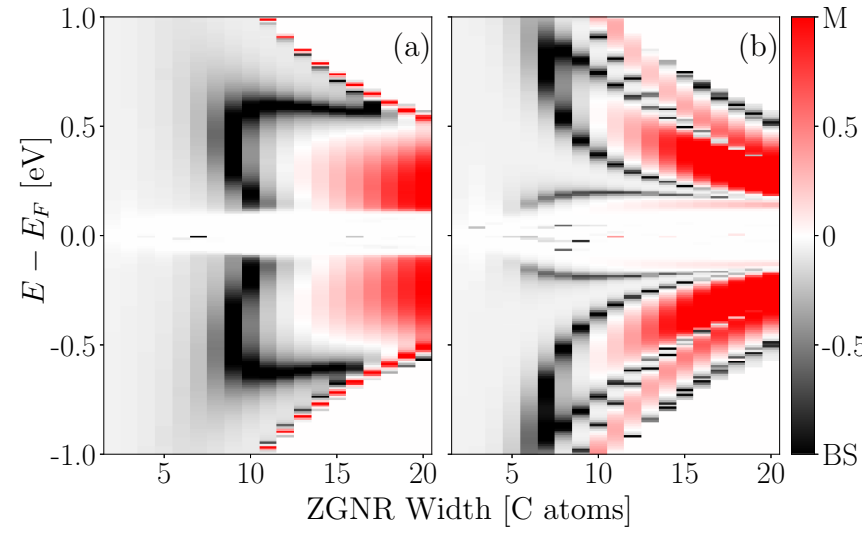

FIG. 5. Figure of merit (FM) for systems composed of ZGNRs in (a) $\mathrm{AB}$ or (b) AA configurations. Black and red regions correspond to situations where a given device is suitable as a BS or M, respectively. White regions are unsuitable as BS or M because of large transmission into the other but the desired output ports.

instance, Fig. 5 reflects that the 8-ZGNR devices shown in Figs. 2(a) and 2(b) are good candidates for BS, consistent with the qualitative picture of Figs. 3(a), 3(b) and 4.

For both $\mathrm{AA}$ and $\mathrm{AB}$ ZGNR devices the transmitted electron wave to the other ribbon is always bent $120^{\circ}$ into electrode 3 (see also the full transmission matrices in Figs. 5

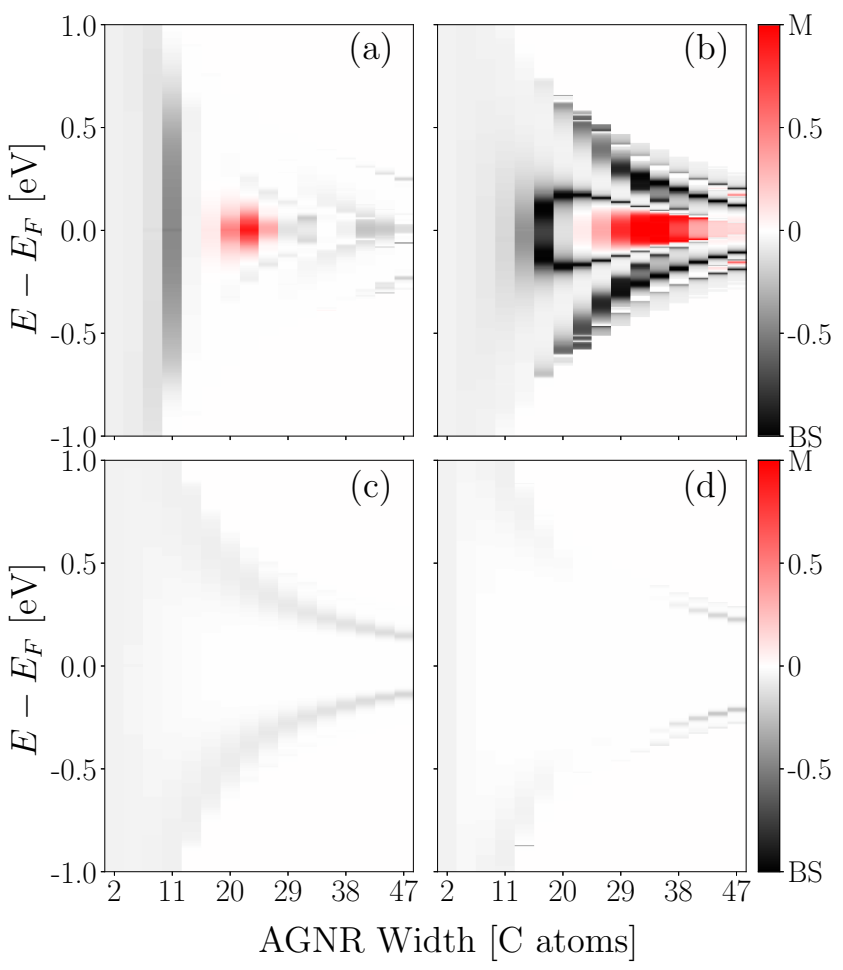

FIG. 6. Figure of merit (FM) for systems composed of AGNRs of the $3 p+2$ family with (a) AA-1, (b) AA-2, (c) AB-1, and (d) AB2 configurations. Black and red regions correspond to situations where a given device is suitable as a BS or M, respectively. White regions are unsuitable as $\mathrm{BS}$ or $\mathrm{M}$ because of large transmission into the other but the desired output ports. 
and S4 [62]). To obtain a M, where an electron incoming from electrode 1 is almost entirely transferred to electrode 3 , one should choose wider ZGNRs.

For the AGNRs the situation is a little more complex. As discussed in Fig. 2, it is possible to form four different stackings (AA-1, AA-2, AB-1, and AB-2). Further, the band gap of AGNRs is determined by the overall ribbon width $W$, which classifies them into three distinct families $3 p$, $3 p+1$, or $3 p+2$ for integer $p[24,25,27,28]$. This leaves us with 12 different situations, considered in terms of the full transmission matrices in Figs. S5-S16 [62]. We find that the most interesting devices are those built with $(3 p+2)$-AGNRs in the AA-stacked configurations. However, compared with the ZGNRs, the parameter space for desirable devices is more restricted and the losses are generally larger. Independent of width, the $\mathrm{AB}$-stacked configurations lead to scattering of the electron wave into all terminals.

We also note here that the qualitative difference mentioned in Sec. III A between the $60^{\circ}$ turn of the transferred electron wave for AA-1 configuration versus the $120^{\circ}$ turn for AA2 is a robust feature across the different families (Figs. S5S16 [62]). Additionally, we also find very thin white regions that do not correspond to high losses but to $T_{12} \sim 1$, immersed in red-e.g., seen for $W=10-15$ in Fig. 5(b) and for $W>20$ in Fig. 6(b). This suggests that crossed GNRs can also work as energy filters. These $T_{12}\left(T_{13}\right)$ peaks (dips), also plotted in Fig. S18 for clarification, become narrower as the width of the ribbons grows, which enhances the energy selection.

\section{E. Robustness of the discussed properties}

So far we have discussed the different transport properties that can be found in the ideal case, that is commensurate GNRs (AA or AB stacking) with a relative angle of $\theta=60^{\circ}$. However, precise control of the device geometry is likely a significant experimental challenge. In this section we therefore proceed to test the robustness of the transport properties against some perturbations of this ideal situation. More specifically, we explore now the exact roles of the intersection angle, deviations from the idealized stacking pattern, lattice deformations via uniaxial strain, variation of the inter-GNR separation, and electrostatic potential differences between the two ribbons.

Since we concluded above that ZGNR devices may be the best candidates for building electron quantum optical setups, we will focus the following discussion around them. We take as the reference device the crossing of two AB-stacked 8ZGNRs [Fig. 2(a)] and compute the transmission probabilities from terminals $\alpha=1$ to $\beta=1,2,3,4$ for each of the above-mentioned perturbations. The AA-stacked 8-ZGNRs were found to display qualitatively similar trends as can be seen from Figs. S19-S23 [62]. We will see that the low-loss property of these devices is thus preserved for the applied variations and in some cases the FM is even significantly enhanced, indicating that almost perfect BS or M could be obtained by tuning the above-mentioned parameters.

\section{Intersection angle}

We first discuss the effect of small rotations of the on-top ribbon starting from the ideal configuration where $\theta=60^{\circ}$. For instance, the twisting angle between the ribbons introduces separated domains of weakly and strongly coupled atoms in the crossing area that might affect the transport properties of these junctions [68]. To isolate the effect of the intersection angle from that of the precise stacking pattern (translation), we apply the rotation around the center of the scattering region (crossing) indicated with a black dot in Fig. 7(a). This ensures that the center of the junction remains unchanged and the effect of the rotation angle perturbs mostly the edge zones of the crossing.

Figure 8 shows the reflection and transmission probabilities for varying angles $\delta \theta= \pm 2^{\circ}$. The results for the reference case of $\theta=60^{\circ}$ are shown as black lines in all panels. We first note that the reflection probability $R_{1}$ does not vary much from its initial value $\sim 0$. The same holds for the (unwanted) transmission $T_{14}$. The main effect is the precise distribution between the transmissions $T_{12}$ and $T_{13}$.

This shows that the angle can be a physical knob to tune the transmission ratio between the two outgoing terminals of a BS. On the other hand, the approximate particle-hole symmetry found for the ideal AB or AA stacking goes away as the lattice mismatch grows. The reflection plane shown in Fig. 2(a) is also lost for $\delta \theta \neq 0$ (and other geometrical distortions), however, we still identify only four qualitative independent elements in $\mathbf{T}$ for all cases.

\section{Lateral translations}

To study the precise lattice matching in the crossing area, we performed a series of calculations where the top GNR is translated by $\Delta_{x}$ along the $x$ axis with respect to the bottom GNR [see Fig. 7(b)]. Due to periodicity it is sufficient to consider translation vectors with modulus $\Delta_{x} \leqslant 2 a \sin \left(60^{\circ}\right) \approx$ $2.46 \AA$.

Figure 9 shows the reflection and transmission probabilities as a function of such translations. Again, the results for the ideal $\mathrm{AB}$ stacking are shown as black lines. As for small variations in the intersection angle, even though this geometrical distortion also intensifies the particle-hole asymmetry as the system goes away from the ideal stacking, $R_{1}$ and $T_{14}$ remain rather unaffected by translation. In other words, the low-loss situation of these devices is robust with respect to translations. On the other hand, the inter-ribbon transfer process of electrons becomes mostly less effective. We interpret this as due to an overall elongation of interlayer atom distances. For this reason $T_{13}$ slightly decreases with the translating of the on-top ribbon, while $T_{12}$ slightly increases with respect to the reference curves (black lines) for most of the cases.

\section{Uniaxial strain}

For experimentally grown GNRs it is relevant to consider the strain-induced deformations, e.g., a lattice mismatch with the supporting substrate [69]. But strain can also be applied in a controlled way [70], for example, using a piezoelectric substrate for shrinking or elongating samples by applying a bias voltage [71]. In these directions we study here a simplified scenario of applying the same uniaxial strain $\varepsilon$ to both GNRs in the device as defined in Fig. 7(c). As explained in the case of variation of the intersecting angle, to isolate the effect of strain on the transport properties of the device, we apply 

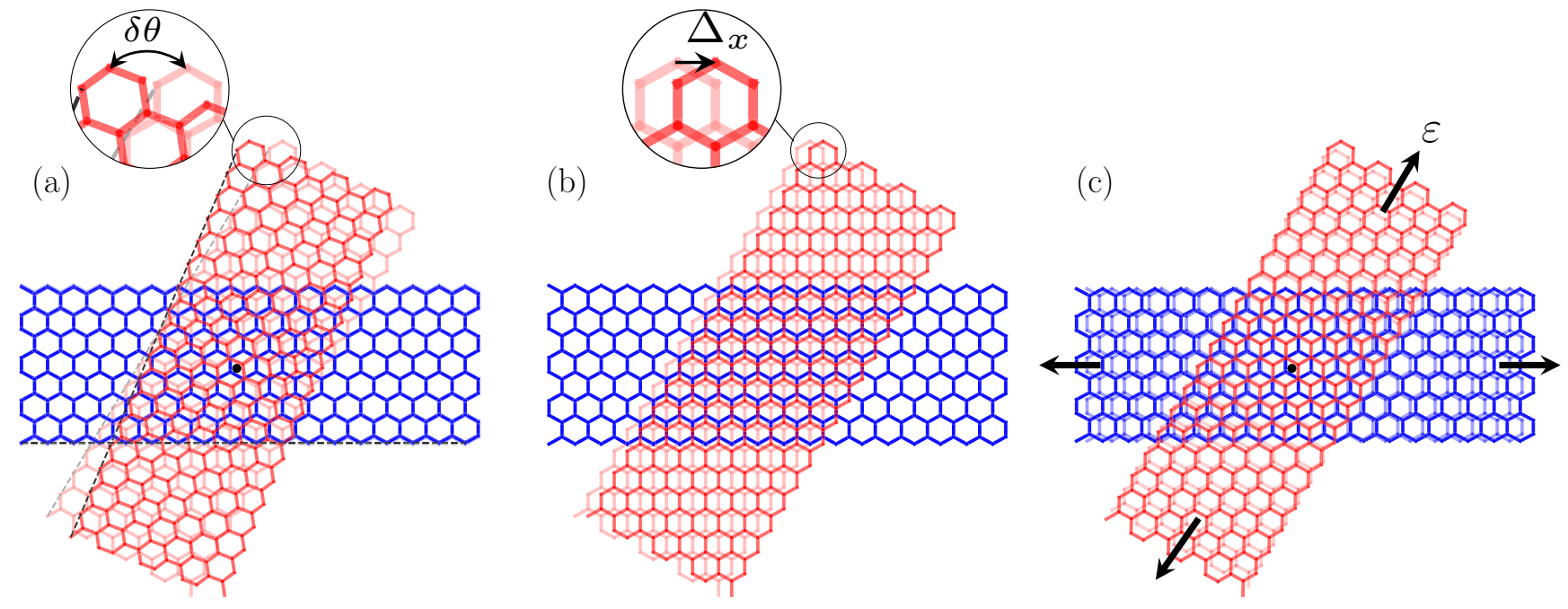

FIG. 7. Sketch of the geometrical distortions applied to the AB-stacked 8-ZGNR device. (a) Rotation by some small angle $\delta \theta$ around the configuration with a relative angle of $\theta=60^{\circ}$. The rotation is performed around the center of the scattering region, indicated by a black dot. (b) Translation of the on-top ribbon with respect to the lower one by an amount $\Delta_{x}$ along the $x$ axis. (c) Strain $\varepsilon$ is applied along the periodic direction of each ribbon while keeping the center of the scattering (black dot) region unchanged.

the strain with respect to the center of the crossing area [as depicted in Fig. 7(c)]. Otherwise arbitrary lattice mismatches could further modify the transmission curves. The main effect of uniaxial strain is that it induces an anisotropy between the atomic bonds and therefore in the electronic structure of the individual GNRs. Additionally, a strain induces some mismatch of the lattices in the crossing region, and therefore changes the scattering potential. The transport properties of the devices are therefore expected to be sensitive to strain. Figure 10 explores uniaxial strain in the range from $-1 \%$ (compression) to $1 \%$ (stretching). Again, both $R_{1}$ and $T_{14}$ are not affected by the lattice deformation, and remain very close to zero in the single-channel energy region.

Looking at the intra- and inter-transmissions $T_{12}$ and $T_{13}$, the curves vary smoothly around the reference values (black lines). The effects of compression and stretching of the GNRs are quite different: GNR compression causes $T_{12}\left(T_{13}\right)$ to increase (decrease), while stretching has the opposite effect. Again, strain can be seen as a physical knob to engineer the device properties. For instance, a strain of $\varepsilon \sim 1 \%$ brings the system closer to the ideal BS with $T_{12}=T_{13}=50 \%$, while keeping both $R_{1} \sim T_{14} \sim 0$. In fact, our FM graph of Fig. 10(e) shows a significant enhancement of the performance of the device as a BS when stretching the device.

\section{Interlayer separation}

The exponential distance dependence of electron transport in the tunneling regime suggests that the separation between ribbons may considerably affect the transport properties. Figure 11 shows the reflection and transmission probabilities as a function of the GNR separation $d$ within an interval determined by $\pm 0.15 \AA$ around a typical van der Waals distance $d=3.34 \AA[43,72,73]$ (black lines in all panels). Apart from the flat-band energy region very close to $E=E_{F}$, the loss channels characterized by $R_{1}$ and $T_{14}$ are largely unaffected.
The main effect of varying $d$ is to control the ratio between the intra- and intertransmissions $T_{12}$ and $T_{13}$, which varies smoothly to almost 0:1 as the ribbon separation $d$ is decreased. In the other direction, the ratio goes (unsurprisingly) to 1:0 as the ribbon separation is increased and therefore eventually become decoupled.

The strong variation with the inter-GNR separation suggests that this is a key parameter to tune the transport properties. An ideal 50:50 BS may thus be obtained by applying an external force to the junction for $d \sim 3.30 \AA$, while a perfect $\mathrm{M}$ is found for $d<3.20 \AA$, as seen in Fig. 11(e), where the plateaus at $\mathrm{FM}=1$ show this behavior. The possibility to use such electromechanical switching has been also proposed to be used for suspended multilayer graphene [74], crossed AGNRs [43], and crossed carbon nanotubes [75].

\section{Electrostatic potential differences}

Here, we discuss the effect of an electrostatic potential difference between the two ribbons. This could, for instance, correspond to an experimental situation where a bias voltage is applied to the GNR electrodes. We consider a potential difference $V$ that modifies uniformly the on-site energies to $\epsilon_{i}-E_{F}=-V / 2$ (and consequently the chemical potentials of the electrodes) of the top (red) ribbon and $\epsilon_{i}-E_{F}=V / 2$ of the bottom (blue) ribbon (see Fig. 1).

Figure 12 shows the reflection and transmission probabilities for the range $|V| \leqslant 0.5 \mathrm{~V}$. Drastic changes are observed in the energy range between the electrode chemical potentials $[-V / 2, V / 2]$, where valence bands (VBs) and conduction bands (CBs) of the two GNRs now overlap. In fact, the mixing of VBs and CBs leads to an interchange of the propagation direction: A transferred electron in the bias window turns $60^{\circ}$ instead of $120^{\circ}$. In fact, our FM [Fig. 12(e)] shows that the performance of the device is enhanced in the energy window $\left|E-E_{F}\right| \leqslant V / 2$, compared to the unbiased case (black curve). In contrast, the single-channel energy region slightly 

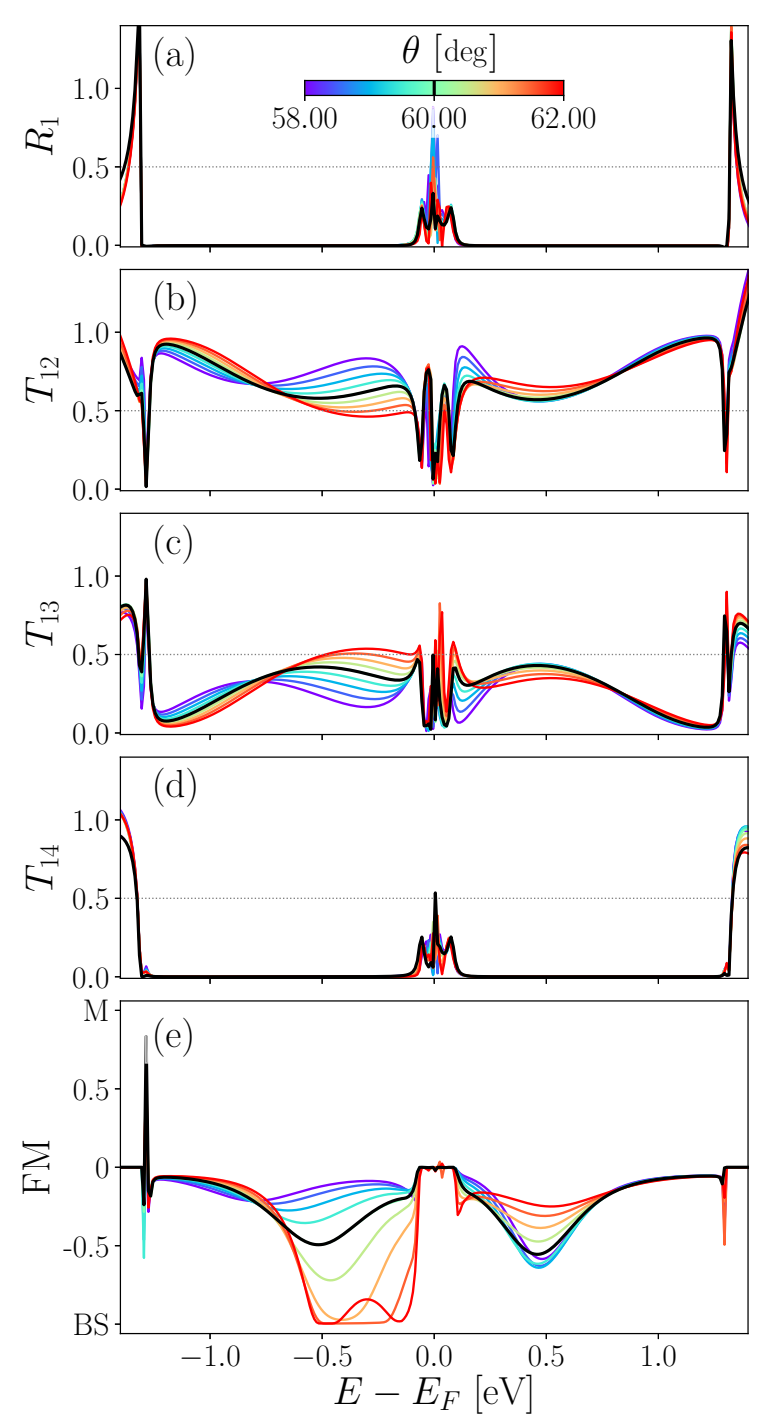

FIG. 8. Variation with respect to the rotation angle between two AB-stacked 8-ZGNRs. Reflection and transmission probabilities, (a) $R_{1}$ (b) $T_{12}$, (c) $T_{13}$, and (d) $T_{14}$, and (e) figure of merit as a function of the incoming electron energy $E-E_{F}$, obtained for different relative angles $(\theta)$ between the ribbons (color lines). The reference probabilities $\left(\theta=60^{\circ}\right)$ are plotted in black solid lines.

shrinks, as the chemical potential shifting produces the edge of the single-mode part of the CB (VB) of the bottom (top) ribbon to overlap with more than one mode in the top (bottom) ribbon. The presence of multiple bands in any of the incoming or outgoing electrodes is responsible for the larger reflection and transmission into the other output, e.g., as it happens for energies $\left|E-E_{F}\right|>1.0 \mathrm{eV}$ in Figs. 12(a) and 12(d).

Outside the bias window the curves are hardly changed, reflecting a low variability of the transport properties even when the elastically transferred electron wave to the other ribbon is now propagating with a different momentum due to the energy offsets of their band structures.

\section{CONCLUSIONS AND OUTLOOK}

In this paper we studied the electronic transport properties of four-terminal devices formed by two intersecting GNRs
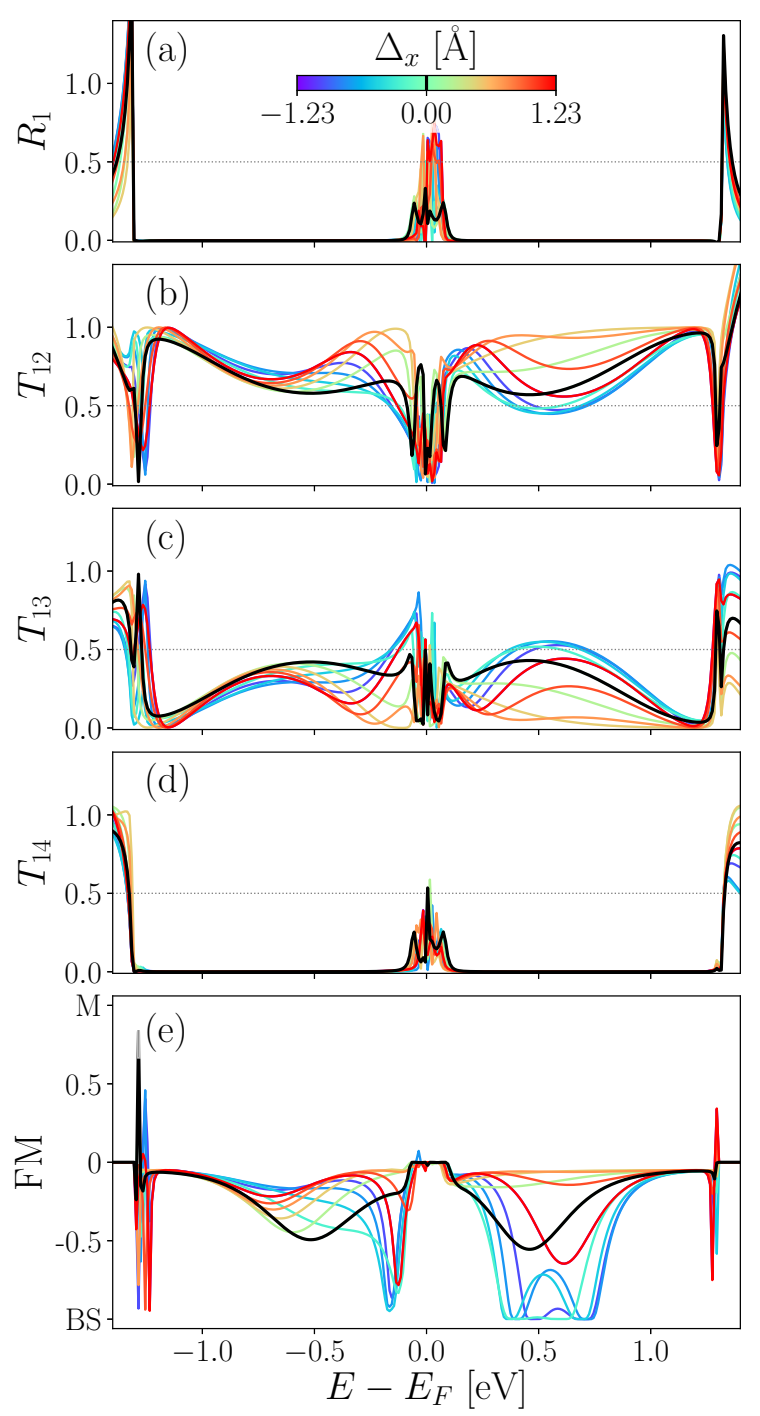

FIG. 9. Variation with respect to the relative lateral displacement between two AB-stacked 8-ZGNRs. Reflection and transmission probabilities, (a) $R_{1}$ (b) $T_{12}$, (c) $T_{13}$, and (d) $T_{14}$, and (e) figure of merit as a function of electron energy $E-E_{F}$, obtained for different translation distances along the $x$ axis $\left(\Delta_{x}\right)$ of the on-top ribbon (color lines). The reference probabilities $\left(\Delta_{x}=0\right)$ are plotted in black solid lines.

with a nominal crossing angle of $\theta=60^{\circ}$. We presented a complete classification and characterization of the different functionalities that can be found in these type of junctions by varying the edge topology of the GNRs (zigzag or armchair), stacking sequence ( $\mathrm{AA}$ or $\mathrm{AB}$ ), width of the ribbons, and energy for the propagating electrons in the valence or conduction bands.

We determined the number of independent transmission probability matrix elements in Eq. (10) that fully characterize their transport behavior: 10,6 , or 4 depending on the degree of symmetry that a given device displays. In practice, however, we found that for low-energy electrons it suffices qualitatively to describe the transmission probabilities with only four independent elements. The reason for this is the fact that the dominant part of the scattering potential contains 

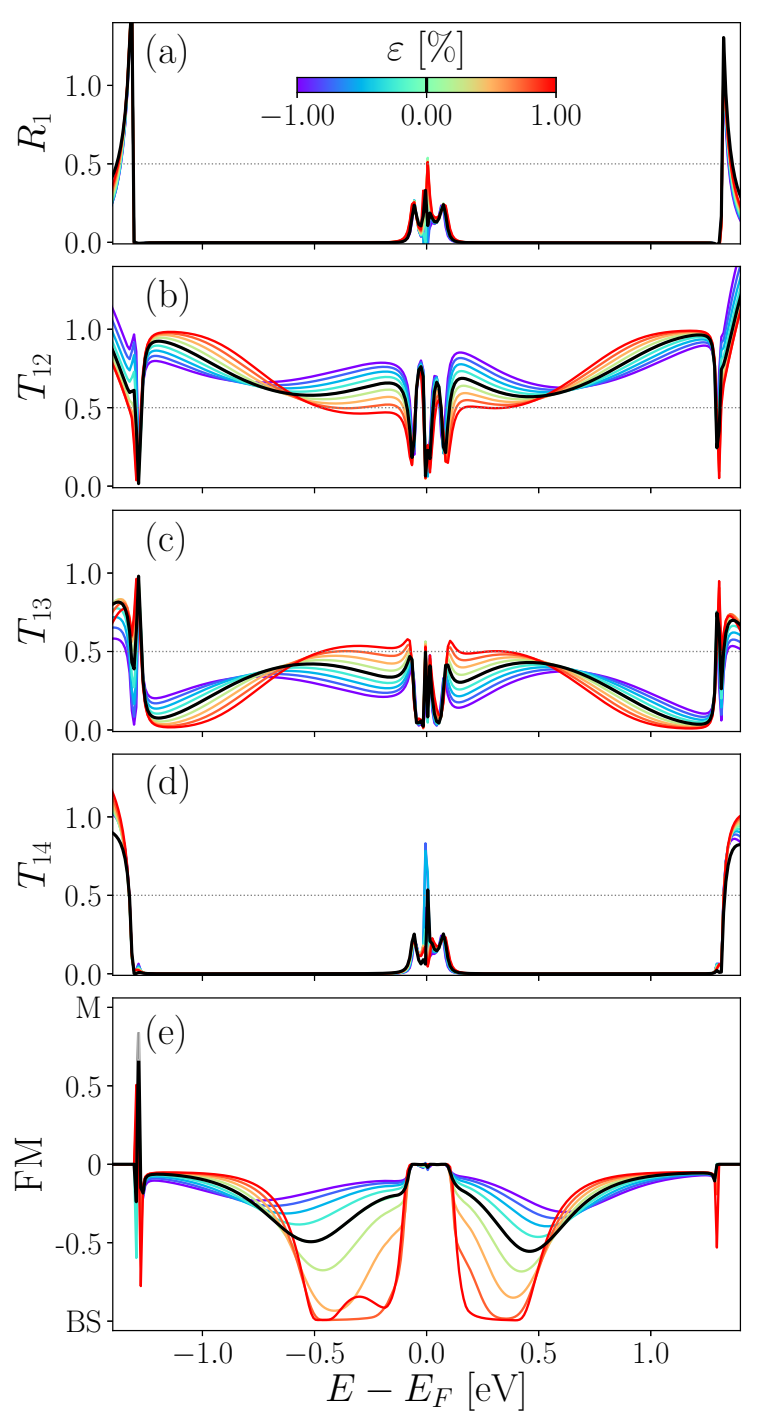

FIG. 10. Variation with respect to the applied uniaxial strain $\varepsilon$ along the periodic direction of each GNR for the two AB-stacked 8-ZGNRs. Reflection and transmission probabilities, (a) $R_{1}$ (b) $T_{12}$, (c) $T_{13}$, and (d) $T_{14}$, and (e) figure of merit as a function of electron energy $E-E_{F}$, obtained for different uniaxial strain $\varepsilon$ applied to both GNRs along the nonconfined direction (color lines). The reference probabilities $(\varepsilon=0)$ are plotted in black solid lines.

more symmetries than that of the device geometry as a whole. Implicitly, this result also means that the strict geometrical symmetry behind the systems is not critical for the GNR crossings to function as beam splitters.

Besides the BS property, we also identified other interesting electron quantum optical functionalities of these devices. For instance, depending on the GNR width and electron energy the device can also behave as a mirror or an energy filter.

Interestingly, for AA-stacked AGNRs we discovered that there exist two different configurations (AA-1 and AA-2) that show little geometrical difference but behave very differently from each other in terms of the electron transport for lowenergy electrons. In the particular case of $3 p+2$-AGNR crossings, the electron beam is only allowed to turn $60^{\circ}$ for
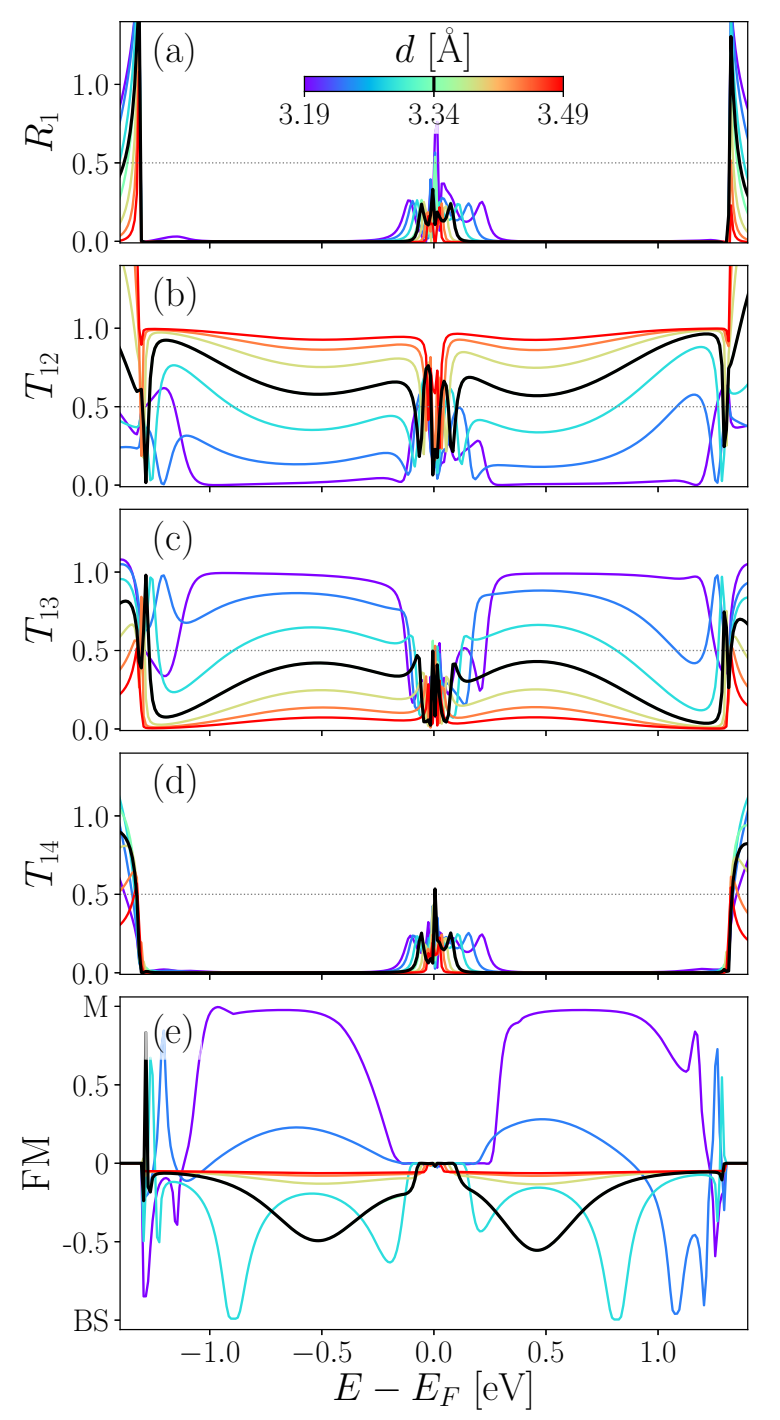

FIG. 11. Variation with respect to the inter-GNR separation of two AB-stacked 8-ZGNRs. Reflection and transmission probabilities, (a) $R_{1}$ (b) $T_{12}$, (c) $T_{13}$, and (d) $T_{14}$, and (e) figure of merit as a function of electron energy $E-E_{F}$ and inter-GNR separation $d$ (color lines). The reference probabilities $(d=3.34 \AA)$ are plotted in black solid lines.

the AA-1 configuration, as opposed to $120^{\circ}$ for the AA-2 configuration. On the other hand, AB-stacked AGNR devices do not show good electron quantum optical features due to the comparatively larger losses and low inter-GNR transmission. Unfortunately, AA-stacked configurations are probably harder to realize in practice (not the most stable energetically) compared to the AB-stacked one [76]. Combined with a generally larger variability of the AGNR transport behavior, these facts indicate that ZGNRs are more interesting objects for the considered device applications than AGNRs.

We further tested the robustness of the predicted transport properties by studying small variations on the intersecting angle between the ribbons, lattice matching in the crossing area, uniaxial strain, interlayer separation, and finite potential differences for devices composed of 8-ZGNRs. While the overall qualitative behavior was found to be robust under 

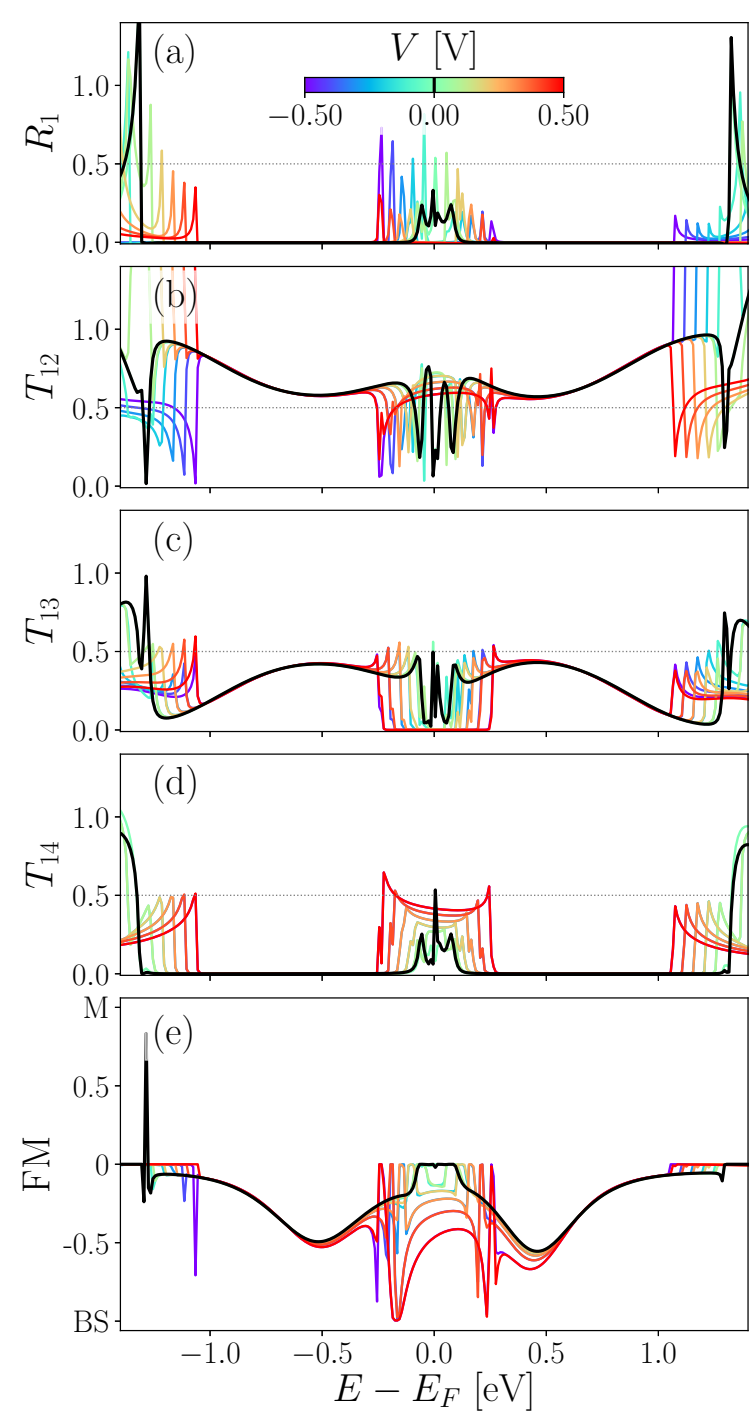

FIG. 12. Variation with respect to potential differences $V$ between the two AB-stacked 8-ZGNRs. Reflection and transmission probabilities, (a) $R_{1}$ (b) $T_{12}$, (c) $T_{13}$, and (d) $T_{14}$, and (e) figure of merit as a function of electron energy $E-E_{F}$, obtained for different values of $V$ (colored lines). The reference probabilities $(V=0)$ are plotted in black solid lines.

these modifications, a strong quantitative response can be obtained-indicating the need to control these effects as well as their potential for tuning the crossed-GNR devices. On the other hand, in this work we considered the situation of a spindegenerate electronic structure. However, ZGNRs have been predicted to develop spin-polarized states localized along the edges of the ribbons close to the Fermi level [24]. This suggests that additional spin-dependent effects could emerge in these devices. The interplay with the physics discussed here could become an interesting topic for future research.

For electron quantum optics applications, the central feature of the considered devices is that they coherently distribute incoming electrons in the intended output ports. In our model, with a precisely given unitary scattering matrix and without considering environmental degrees of freedom, all the considered devices process the input coherently. The analysis of the operative decoherence processes in GNR-based devices is an important task for future work. In particular, a single purestate electron injected into one arm of a BS device discussed here is mapped to an (mode-)entangled state of the output ports. Such entanglement could be verified experimentally, for example, by measuring the state's Bell correlations as discussed in Ref. [13]. A second basic application of the BS device is the Hanbury Brown-Twiss setup [8-11], which can be used to study the indistinguishability of electrons prepared in different input ports by the observation of antibunching in the output ports of the BS. A theoretical analysis of these experiments would include the investigation of the influence of environmental degrees of freedom (phonons, electrons in the substrate, or fluctuating perturbations such as the ones discussed in Sec. IIIE), and, in the case of the Hanbury Brown-Twiss setup, also the effect of the interaction between electrons in the BS. An important prerequisite for all such experiments are methods to inject single electrons in a welldefined mode and to reliably detect them.

Finally, we envision that the functionalities presented here may be interesting as fundamental building blocks in larger electronic networks based on GNRs. For instance, with four GNRs one could construct the electronic analog of the MachZehnder interferometer, consisting of two beam splitters and two oriented mirrors at the intersection of pairwise parallel ribbons. Such a versatile setup, sensitive to the relative phase shift between two spatially separated pathways, has a wide range of quantum technology applications, e.g., metrology, entanglement, cryptography, and information processing [18].

\section{ACKNOWLEDGMENTS}

This work was supported by the project Spanish Ministerio de Economía y Competitividad (MINECO) through the Grants no. FIS2017-83780-P (Graphene Nanostructures "GRANAS") and no.MAT2016-78293-C6-4R, the Basque Departamento de Educación through the PhD fellowship no. PRE_2019_2_0218 (S.S.), the University of the Basque Country through the Grant no. IT1246-19, and the European Union (EU) through Horizon 2020 (FET-Open project SPRING Grant no. 863098).

\section{APPENDIX: COMPARISON WITH DFT CALCULATIONS}

In this Appendix we compare the results presented in the main text with DFT, another popular theoretical approach used in the field of solid state physics. In particular, we choose to compute the electronic structure of AB-stacked bilayer graphene as a model system to establish suitable parameters for the general TB Hamiltonian introduced in Sec. II. We further simulate the electron transport characteristics of the specific device geometries shown in Fig. 2 for detailed benchmarking.

We employ the self-consistent DFT and NEGF methods as implemented in the SIESTA/TRANSIESTA [55,61,77] packages. All calculations of this kind used the van der Waals (vdW) density functional [78] with the modified exchange by Klimeš et al. [79]. The core electrons were described with Troullier-Martins pseudopotentials [80] and a double- $\zeta$ basis set defined with a $30 \mathrm{meV}$ energy shift was used to 


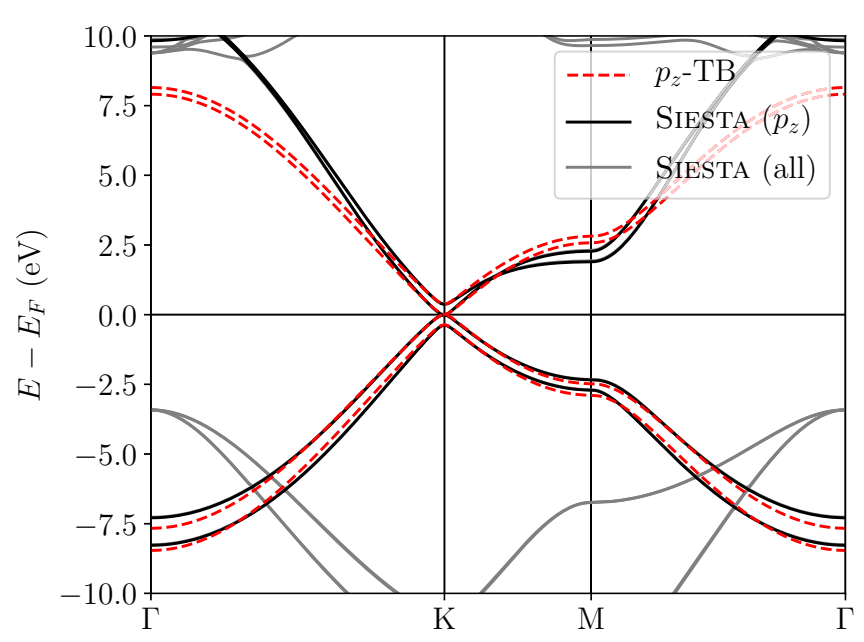

FIG. 13. Band structure of AB-stacked bilayer graphene along the $\Gamma-K-M-\Gamma$ path of the Brillouin zone, obtained with DFT (black and gray solid lines) and TB methods (red dashed lines), with the fitted hopping parameters described in the text. The bond length is set to $a=1.42 \AA$ and the interlayer separation to $d=3.34 \AA$. Black lines correspond to the graphene $\pi$ bands (formed by the $p_{z}$ orbitals) while the gray lines show the graphene $\sigma$ bands absent in the TB model.

expand the valence-electron wave functions. The fineness of the real-space integration mesh was defined using a $350 \mathrm{Ry}$ energy cutoff. All carbon atoms were saturated at the edges with hydrogen atoms.

Figure 13 shows the calculated electronic bands along the $\Gamma-K-M-\Gamma$ path of the Brillouin zone of AB-stacked bilayer graphene obtained with SIESTA [55]. Given the usage of a double- $\zeta$ basis set, the orthogonal $\sigma$ and $\pi$ bands have simple representations in terms of the $\left\{s, p_{x}, p_{y}\right\}$ and $\left\{p_{z}\right\}$ basis orbitals, respectively. To map the DFT electronic structure onto the effective TB model in Eqs. (2)-(5), it is thus sufficient to consider only the $p_{z}$ part of the DFT Hamiltonian. Since we are interested in the low-energy physics, we fitted the TB bands inside an energy window of $\left|E-E_{F}\right| \leqslant 2 \mathrm{eV}$ using nonlinear least squares and obtained the following optimal hopping parameters used in the main text: $t_{\|}=2.682 \mathrm{eV}$, $t^{\prime}=2.7 \mathrm{meV}$, and $t_{\perp}=0.371 \mathrm{eV}$. The corresponding $\mathrm{TB}$ bands with these parameters are plotted in red dashed lines in Fig. 13, showing a very good agreement in the energy range of relevance in this work. Albeit unnecessary for the purposes here, we note that the significant deviations at the $\pi$ band edges can readily be improved with a nonorthogonal TB
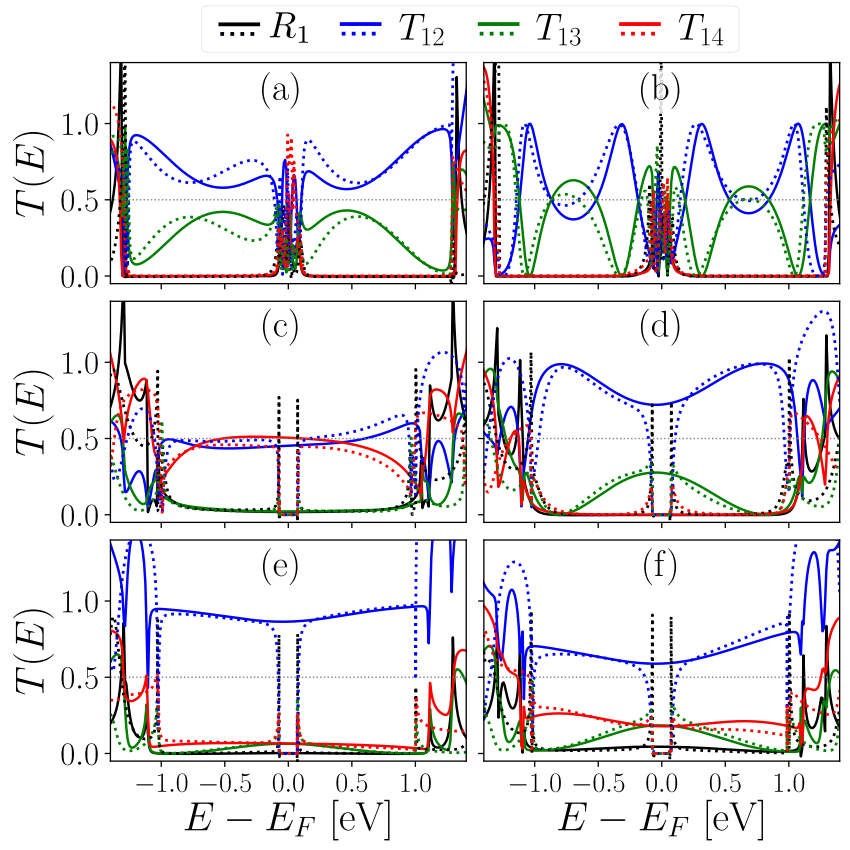

FIG. 14. Reflection and transmission probabilities $R_{1}$ (black), $T_{12}$ (blue), $T_{13}$ (green), and $T_{14}$ (red), obtained with both TB (solid lines) and DFT (dotted lines) methods, through the devices of Fig. 2: two crossed 8-ZGNRs in configuration (a) $\mathrm{AB}$ and (b) AA, and two crossed 11-AGNRs in configuration (c) AA-1, (d) AA-2, (e) AB-1, and (f) AB-2.

model by introduction of additional parameters for the overlap matrix.

Having fixed the parameters for the TB model, we proceed to compare it against the derived transport properties from DFT-NEGF for the six characteristic devices shown Fig. 2. Figure 14 shows the computed reflection and transmission probabilities from TB (solid lines) and DFT (dotted lines) within an energy window of $\left|E-E_{F}\right| \lesssim 1.5 \mathrm{eV}$. Apart from different magnitudes of the AGNR band gap (known to be related to edge effects ignored in this TB modeling [25]), the two models only show minor numerical differences. Overall, the two models provide very similar shapes and quantitative results for the transmission functions. From Figs. 13 and 14 we therefore conclude that the TB method used in the main text provides an accurate description of the essential physics in the energy range we are interested in here.
[1] J. Splettstoesser and R. J. Haug, Special issue: Single-electron control in solid state devices, Phys. Status Solidi B 254, 1770213 (2017).

[2] C. Bäuerle, D. C. Glattli, T. Meunier, F. Portier, P. Roche, P. Roulleau, S. Takada, and X. Waintal, Coherent control of single electrons: A review of current progress, Rep. Prog. Phys. 81, 056503 (2018).

[3] Y. Ji, Y. Chung, D. Sprinzak, M. Heiblum, D. Mahalu, and H. Shtrikman, An electronic Mach-Zehnder interferometer, Nature (London) 422, 415 (2003).
[4] P. Roulleau, F. Portier, D. C. Glattli, P. Roche, A. Cavanna, G. Faini, U. Gennser, and D. Mailly, Finite bias visibility of the electronic Mach-Zehnder interferometer, Phys. Rev. B 76, 161309(R) (2007).

[5] Y. Zhang, D. T. McClure, E. M. Levenson-Falk, C. M. Marcus, L. N. Pfeiffer, and K. W. West, Distinct signatures for Coulomb blockade and Aharonov-Bohm interference in electronic FabryPérot interferometers, Phys. Rev. B 79, 241304(R) (2009).

[6] D. T. McClure, Y. Zhang, B. Rosenow, E. M. Levenson-Falk, C. M. Marcus, L. N. Pfeiffer, and K. W. West, Edge-State 
Velocity and Coherence in a Quantum Hall Fabry-Pérot Interferometer, Phys. Rev. Lett. 103, 206806 (2009).

[7] E. Carbonell-Sanromà, P. Brandimarte, R. Balog, M. Corso, S. Kawai, A. Garcia-Lekue, S. Saito, S. Yamaguchi, E. Meyer, D. Sánchez-Portal et al., Quantum dots embedded in graphene nanoribbons by chemical substitution, Nano Lett. 17, 50 (2017).

[8] M. Henny, S. Oberholzer, C. Strunk, T. Heinzel, K. Ensslin, M. Holland, and C. Schönenberger, The fermionic Hanbury Brown and Twiss experiment, Science 284, 296 (1999).

[9] W. D. Oliver, J. Kim, R. C. Liu, and Y. Yamamoto, Hanbury Brown and Twiss-type experiment with electrons, Science 284, 299 (1999).

[10] P. Samuelsson, E. V. Sukhorukov, and M. Büttiker, Two-Particle Aharonov-Bohm Effect and Entanglement in the Electronic Hanbury Brown-Twiss setup, Phys. Rev. Lett. 92, 026805 (2004).

[11] I. Neder, N. Ofek, Y. Chung, M. Heiblum, D. Mahalu, and V. Umansky, Interference between two indistinguishable electrons from independent sources, Nature (London) 448, 333 (2007).

[12] J. Splettstoesser, P. Samuelsson, M. Moskalets, and M. Büttiker, Two-particle Aharonov-Bohm effect in electronic interferometers, J. Phys. A: Math. Theor. 43, 354027 (2010).

[13] D. Dasenbrook, J. Bowles, J. B. Brask, P. P. Hofer, C. Flindt, and N. Brunner, Single-electron entanglement and nonlocality, New J. Phys. 18, 043036 (2016).

[14] C. H. Bennett, G. Brassard, C. Crépeau, R. Jozsa, A. Peres, and W. K. Wootters, Teleporting an Unknown Quantum State via Dual Classical and Einstein-Podolsky-Rosen Channels, Phys. Rev. Lett. 70, 1895 (1993).

[15] T. Debarba, F. Iemini, G. Giedke, and N. Friis, Teleporting quantum information encoded in fermionic modes, Phys. Rev. A 101, 052326 (2020).

[16] E. Bocquillon, V. Freulon, J.-M. Berroir, P. Degiovanni, B. Plaçais, A. Cavanna, Y. Jin, and G. Fève, Coherence and indistinguishability of single electrons emitted by independent sources, Science 339, 1054 (2013).

[17] V. Giovannetti, D. Frustaglia, F. Taddei, and R. Fazio, Electronic Hong-Ou-Mandel interferometer for multimode entanglement detection, Phys. Rev. B 74, 115315 (2006).

[18] A. Sarkar, T. K. Bhattacharyya, and A. Patwardhan, Quantum logic processor: Implementation with electronic Mach-Zehnder interferometer, Appl. Phys. Lett. 88, 213113 (2006).

[19] P. Rickhaus, P. Makk, M.-H. Liu, K. Richter, and C. Schönenberger, Gate tuneable beamsplitter in ballistic graphene, Appl. Phys. Lett. 107, 251901 (2015).

[20] S. Chen, Z. Han, M. M. Elahi, K. M. M. Habib, L. Wang, B. Wen, Y. Gao, T. Taniguchi, K. Watanabe, J. Hone et al., Electron optics with p-n junctions in ballistic graphene, Science 353, 1522 (2016).

[21] K. Nakada, M. Fujita, G. Dresselhaus, and M. S. Dresselhaus, Edge state in graphene ribbons: Nanometer size effect and edge shape dependence, Phys. Rev. B 54, 17954 (1996).

[22] M. Fujita, K. Wakabayashi, K. Nakada, and K. Kusakabe, Peculiar localized state at zigzag graphite edge, J. Phys. Soc. Jpn. 65, 1920 (1996).

[23] F. Schwierz, Graphene transistors, Nat. Nanotechnol. 5, 487 (2010).

[24] Y.-W. Son, M. L. Cohen, and S. G. Louie, Half-metallic graphene nanoribbons, Nature (London) 444, 347 (2006).
[25] Y.-W. Son, M. L. Cohen, and S. G. Louie, Energy Gaps in Graphene Nanoribbons, Phys. Rev. Lett. 97, 216803 (2006).

[26] M. Y. Han, B. Özyilmaz, Y. Zhang, and P. Kim, Energy BandGap Engineering of Graphene Nanoribbons, Phys. Rev. Lett. 98, 206805 (2007).

[27] L. Yang, C.-H. Park, Y.-W. Son, M. L. Cohen, and S. G. Louie, Quasiparticle Energies and Band Gaps in Graphene Nanoribbons, Phys. Rev. Lett. 99, 186801 (2007).

[28] T. Wassmann, A. P. Seitsonen, A. M. Saitta, M. Lazzeri, and F. Mauri, Structure, Stability, Edge States, and Aromaticity of Graphene Ribbons, Phys. Rev. Lett. 101, 096402 (2008).

[29] S. Minke, J. Bundesmann, D. Weiss, and J. Eroms, Phase coherent transport in graphene nanoribbons and graphene nanoribbon arrays, Phys. Rev. B 86, 155403 (2012).

[30] J. Baringhaus, M. Ruan, F. Edler, A. Tejeda, M. Sicot, A. Taleb-Ibrahimi, A.-P. Li, Z. Jiang, E. H. Conrad, C. Berger et al., Exceptional ballistic transport in epitaxial graphene nanoribbons, Nature (London) 506, 349 (2014).

[31] J. Aprojanz, S. R. Power, P. Bampoulis, S. Roche, A.-P. Jauho, H. J. W. Zandvliet, A. A. Zakharov, and C. Tegenkamp, Ballistic tracks in graphene nanoribbons, Nat. Commun. 9, 4426 (2018).

[32] L. P. Zârbo and B. K. Nikolić, Spatial distribution of local currents of massless Dirac fermions in quantum transport through graphene nanoribbons, Europhys. Lett. 80, 47001 (2007).

[33] J. Cai, P. Ruffieux, R. Jaafar, M. Bieri, T. Braun, S. Blankenburg, M. Muoth, A. P. Seitsonen, M. Saleh, X. Feng et al., Atomically precise bottom-up fabrication of graphene nanoribbons, Nature (London) 466, 470 (2010).

[34] P. Ruffieux, S. Wang, B. Yang, C. Sánchez-Sánchez, J. Liu, T. Dienel, L. Talirz, P. Shinde, C. A. Pignedoli, D. Passerone et al., On-surface synthesis of graphene nanoribbons with zigzag edge topology, Nature (London) 531, 489 (2016).

[35] M. Koch, F. Ample, C. Joachim, and L. Grill, Voltagedependent conductance of a single graphene nanoribbon, Nat. Nanotechnol. 7, 713 (2012).

[36] S. Kawai, A. Benassi, E. Gnecco, H. Söde, R. Pawlak, X. Feng, K. Müllen, D. Passerone, C. A. Pignedoli, P. Ruffieux et al., Superlubricity of graphene nanoribbons on gold surfaces, Science 351, 957 (2016).

[37] D. A. Areshkin and C. T. White, Building blocks for integrated graphene circuits, Nano Lett. 7, 3253 (2007).

[38] T. Jayasekera and J. W. Mintmire, Transport in multiterminal graphene nanodevices, Nanotechnology 18, 424033 (2007).

[39] L. Jiao, L. Zhang, L. Ding, J. Liu, and H. Dai, Aligned graphene nanoribbons and crossbars from unzipped carbon nanotubes, Nano Res. 3, 387 (2010).

[40] B. G. Cook, P. Dignard, and K. Varga, Calculation of electron transport in multiterminal systems using complex absorbing potentials, Phys. Rev. B 83, 205105 (2011).

[41] A. R. Botello-Méndez, E. Cruz-Silva, J. M. Romo-Herrera, F. López-Urías, M. Terrones, B. G. Sumpter, H. Terrones, J.-C. Charlier, and V. Meunier, Quantum transport in graphene nanonetworks, Nano Lett. 11, 3058 (2011).

[42] L. R. F. Lima, A. R. Hernández, F. A. Pinheiro, and C. Lewenkopf, A 50/50 electronic beam splitter in graphene nanoribbons as a building block for electron optics, J. Phys.: Condens. Matter 28, 505303 (2016).

[43] P. Brandimarte, M. Engelund, N. Papior, A. Garcia-Lekue, T. Frederiksen, and D. Sánchez-Portal, A tunable electronic beam 
splitter realized with crossed graphene nanoribbons, J. Chem. Phys. 146, 092318 (2017).

[44] P. R. Wallace, The band theory of graphite, Phys. Rev. 71, 622 (1947).

[45] M. S. Dresselhaus and G. Dresselhaus, Intercalation compounds of graphite, Adv. Phys. 51, 1 (2002).

[46] S. Reich, J. Maultzsch, C. Thomsen, and P. Ordejón, Tightbinding description of graphene, Phys. Rev. B 66, 035412 (2002).

[47] E. McCann and V. I. Fal'ko, Landau-Level Degeneracy and Quantum Hall Effect in a Graphite Bilayer, Phys. Rev. Lett. 96, 086805 (2006).

[48] L. M. Malard, J. Nilsson, D. C. Elias, J. C. Brant, F. Plentz, E. S. Alves, A. H. Castro Neto, and M. A. Pimenta, Probing the electronic structure of bilayer graphene by Raman scattering, Phys. Rev. B 76, 201401(R) (2007).

[49] J. C. Slater and G. F. Koster, Simplified LCAO method for the periodic potential problem, Phys. Rev. 94, 1498 (1954).

[50] J. M. B. Lopes dos Santos, N. M. R. Peres, and A. H. Castro Neto, Graphene Bilayer with a Twist: Electronic Structure, Phys. Rev. Lett. 99, 256802 (2007).

[51] G. Trambly de Laissardière, D. Mayou, and L. Magaud, Localization of Dirac electrons in rotated graphene bilayers, Nano Lett. 10, 804 (2010).

[52] L. Malard, M. Pimenta, G. Dresselhaus, and M. Dresselhaus, Raman spectroscopy in graphene, Phys. Rep. 473, 51 (2009).

[53] A. H. Castro Neto, F. Guinea, N. M. R. Peres, K. S. Novoselov, and A. K. Geim, The electronic properties of graphene, Rev. Mod. Phys. 81, 109 (2009).

[54] F. Guinea and N. R. Walet, Continuum models for twisted bilayer graphene: Effect of lattice deformation and hopping parameters, Phys. Rev. B 99, 205134 (2019).

[55] J. M. Soler, E. Artacho, J. D. Gale, A. García, J. Junquera, P. Ordejón, and D. Sánchez-Portal, The SIESTA method for $a b$ initio order- $N$ materials simulation, J. Phys.: Condens. Matter 14, 2745 (2002).

[56] L. Kadanoff and G. Baym, Quantum Statistical Mechanics: Green's Function Methods in Equilibrium and Nonequilibrium Problems, Frontiers in Physics: A Lecture Note and Reprint Series (W. A. Benjamin, New York, 1962).

[57] L. V. Keldysh, Diagram technique for nonequilibrium processes, Sov. Phys. JETP 20, 1018 (1965) [Zh. Eksp. Theor. Fiz. 47, 1515 (1964)].

[58] H. Haug and A.-P. Jauho, Quantum Kinetics in Transport and Optics of Semiconductors, 2nd ed. (Springer, Berlin, 2007).

[59] M. Büttiker, Y. Imry, R. Landauer, and S. Pinhas, Generalized many-channel conductance formula with application to small rings, Phys. Rev. B 31, 6207 (1985).

[60] N. R. Papior, SISL version 0.9.8 10.5281/zenodo.3661117 (2020).

[61] N. Papior, N. Lorente, T. Frederiksen, A. García, and M. Brandbyge, Improvements on non-equilibrium and transport Green function techniques: The next-generation TranSIESTA, Comput. Phys. Commun. 212, 8 (2017).

[62] See Supplemental Material at http://link.aps.org/supplemental/ 10.1103/PhysRevB.102.035436 for the transport properties of the remaining devices (AA-stacked ZGNRs and crossed
AGNRs), a detailed version of each crossing's scattering potential, and other complementary simulations.

[63] G. Li, A. Luican, J. M. B. Lopes dos Santos, A. H. Castro Neto, A. Reina, J. Kong, and E. Y. Andrei, Observation of van hove singularities in twisted graphene layers, Nat. Phys. 6, 109 (2010).

[64] Y. Cao, V. Fatemi, A. Demir, S. Fang, S. L. Tomarken, J. Y. Luo, J. D. Sanchez-Yamagishi, K. Watanabe, T. Taniguchi, E. Kaxiras et al., Correlated insulator behaviour at half-filling in magic-angle graphene superlattices, Nature (London) 556, 80 (2018).

[65] Y. Cao, V. Fatemi, S. Fang, K. Watanabe, T. Taniguchi, E. Kaxiras, and P. Jarillo-Herrero, Unconventional superconductivity in magic-angle graphene superlattices, Nature (London) 556, 43 (2018).

[66] L. Brey and H. A. Fertig, Electronic states of graphene nanoribbons studied with the Dirac equation, Phys. Rev. B 73, 235411 (2006).

[67] K. Wakabayashi, Y. Takane, and M. Sigrist, Perfectly Conducting Channel and Universality Crossover in Disordered Graphene Nanoribbons, Phys. Rev. Lett. 99, 036601 (2007).

[68] H. M. Abdullah, B. V. Duppen, M. Zarenia, H. Bahlouli, and F. M. Peeters, Quantum transport across van der Waals domain walls in bilayer graphene, J. Phys.: Condens. Matter 29, 425303 (2017).

[69] Z. H. Ni, W. Chen, X. F. Fan, J. L. Kuo, T. Yu, A. T. S. Wee, and Z. X. Shen, Raman spectroscopy of epitaxial graphene on a SiC substrate, Phys. Rev. B 77, 115416 (2008).

[70] C. Si, Z. Sun, and F. Liu, Strain engineering of graphene: A review, Nanoscale 8, 3207 (2016).

[71] F. Ding, H. Ji, Y. Chen, A. Herklotz, K. Dörr, Y. Mei, A. Rastelli, and O. G. Schmidt, Stretchable graphene: A close look at fundamental parameters through biaxial straining, Nano Lett. 10, 3453 (2010).

[72] Y. Baskin and L. Meyer, Lattice constants of graphite at low temperatures, Phys. Rev. 100, 544 (1955).

[73] Y. X. Zhao and I. L. Spain, X-ray diffraction data for graphite to $20 \mathrm{GPa}$, Phys. Rev. B 40, 993 (1989).

[74] Z. Shi, H. Lu, L. Zhang, R. Yang, Y. Wang, D. Liu, H. Guo, D. Shi, H. Gao, E. Wang et al., Studies of graphene-based nanoelectromechanical switches, Nano Res. 5, 82 (2012).

[75] Y.-G. Yoon, M. S. C. Mazzoni, H. J. Choi, J. Ihm, and S. G. Louie, Structural Deformation and Intertube Conductance of Crossed Carbon Nanotube Junctions, Phys. Rev. Lett. 86, 688 (2001).

[76] E. Mostaani, N. D. Drummond, and V. I. Fal'ko, Quantum Monte Carlo Calculation of the Binding Energy of Bilayer Graphene, Phys. Rev. Lett. 115, 115501 (2015).

[77] M. Brandbyge, J.-L. Mozos, P. Ordejón, J. Taylor, and K. Stokbro, Density-functional method for nonequilibrium electron transport, Phys. Rev. B 65, 165401 (2002).

[78] M. Dion, H. Rydberg, E. Schröder, D. C. Langreth, and B. I. Lundqvist, Van der Waals Density Functional for General Geometries, Phys. Rev. Lett. 92, 246401 (2004).

[79] J. Klimeš, D. R. Bowler, and A. Michaelides, Chemical accuracy for the van der Waals density functional, J. Phys.: Condens. Matter 22, 022201 (2009).

[80] N. Troullier and J. L. Martins, Efficient pseudopotentials for plane-wave calculations, Phys. Rev. B 43, 1993 (1991). 